\title{
Research Article \\ Systematic Analysis of the Oncogenic Role of WDR62 in Human Tumors
}

\author{
Yulan Bu, ${ }^{1,2}$ Lihua Zhang, ${ }^{2}$ Xiaolin Ma, ${ }^{3}$ Rui Wang, ${ }^{1,3}$ Xuecheng Zhang $\mathbb{D},{ }^{2}$ and Jiaqiu Li $\mathbb{D}, 3$ \\ ${ }^{1}$ Jinming Yu Academician Workstation of Oncology, Clinical Research Center, Affiliated Hospital of Weifang Medical University, \\ Weifang 261031, China \\ ${ }^{2}$ Department of Radiotherapy, Affiliated Hospital of Weifang Medical University, Weifang 261031, China \\ ${ }^{3}$ Department of Oncology, Affiliated Hospital of Weifang Medical University, Weifang 261031, China
}

Correspondence should be addressed to Xuecheng Zhang; zhangxuechengwf@163.com and Jiaqiu Li; lijq@wfmc.edu.cn

Yulan $\mathrm{Bu}$ and Lihua Zhang contributed equally to this work.

Received 13 March 2021; Revised 2 June 2021; Accepted 8 June 2021; Published 2 July 2021

Academic Editor: Heng Zhou

Copyright @ 2021 Yulan Bu et al. This is an open access article distributed under the Creative Commons Attribution License, which permits unrestricted use, distribution, and reproduction in any medium, provided the original work is properly cited.

Background. Emerging studies support the oncogenic role of WD repeat domain 62 (WDR62) in few tumors, while no pan-cancer analysis is available. In this study, we analyzed systematically the oncogenic role of WDR62 across a series of human tumors based on bioinformatic data mining. Methods. The expression level of WDR62 was analyzed via GEPIA2, TIMER, UALCAN, and StarBase databases. The prognostic role was analyzed via GEPIA2, TIMER, UALCAN, StarBase, TISIDB, TCGA portal, KaplanMeier Plotter, and PrognoScan databases. Then, we explored the causes for WDR62 abnormal expression via TCGA portal and UALCAN databases. Subsequently, the STRING and GeneMANIA databases were used to find the interactive networks for WDR62. Furthermore, we analyzed the correlation between WDR62 expression and immune features via TIMER and TISIDB databases. Results. We found that WDR62 was significantly upregulated in most of the tumors and correlated with poor prognosis mainly in 6 candidate tumors-BLCA, BRCA, KIRC, KIRP, LIHC, and LUAD. Abnormal WDR62 expression may be probably attributed to TP53 mutation and promoter DNA methylation. Relative network analysis demonstrated that WDR62 was mainly involved in MAPK and toll-like receptor signaling pathway. WDR62 expression was associated with various immune cell infiltrations, especially cancer-associated fibroblasts (CAF) and T cell regulatory (Treg) cells, and was markedly correlated with poor prognosis. Moreover, WDR62 expression was closely associated with the expression of some immunomodulators such as PDL1 and has a significant prognostic value. Conclusions. Our study revealed that WDR62 could serve as a diagnostic and prognostic biomarker for several cancers. Importantly, WDR62 was closely associated with various immune cell infiltration, and to a certain extent, it can predict the effect of immunotherapy in particular PD1/PD-L1 inhibitors. Our pan-cancer study provided useful information on the oncogenic role of WDR62, contributing to further exploring the underlying mechanisms.

\section{Introduction}

According to the latest statistics report released by the International Agency for Research on Cancer (IARC), an estimate is approximately 19.3 million new cancer cases and 10.0 million cancer deaths in 2020 worldwide [1]. Among them, the incidence rate of breast cancer has surpassed that of lung cancer as the most diagnosed tumor for the first time. Despite great progress in cancer diagnosis and treatment, it is still not satisfactory in consideration of its leading threat to human life. Therefore, efforts to explore novel biomarkers for the diagnosis of cancers and the prediction of cancer therapeutic effect are critical for cancer control.

With the development of high-throughput sequencing technology, a lot of databases especially those based on TCGA (The Cancer Genome Atlas) and GEO (Gene Expression Omnibus) datasets have emerged [2,3]. Consequently, it is convenient for us to conduct a pan-cancer analysis of genes 
of interest, promoting the discovery of new biomarkers. Targeting immune checkpoint, in particular PD1/PD-L1 inhibitors, has shown clinical activity in a wide spectrum of tumors such as lung cancer [4]. However, as an evolving treatment, PD1/PD-L1 inhibitors only benefited a limited number of patients, demonstrating the complexity of tumorigenesis. It is essential for the establishment of new biomarkers to identify patients who are suitable for immunotherapy. Meanwhile, some biomarkers may predict clinical response and account for the underlying resistance mechanism, providing guidance for immunotherapy in the clinic [5]. WDR62, a novel biomarker, is known to be responsible for the carcinogenesis of few tumors such as ovarian cancer and gastric cancer [6, 7]. These findings indicated that WDR62 overexpression was correlated with centrosome amplification and was confirmed as a novel prognostic biomarker. However, the oncogenic role of WDR62 in other cancers remains unknown. This study is aimed at analyzing systematically the oncogenic role of WDR62 across a series of human tumors based on bioinformatic data mining. Furthermore, the association between WDR62 expression and immune features was investigated in our study for the first time.

\section{Materials and Methods}

2.1. GEPIA2 Database Analysis. We used the "Box Plot" module of the GEPIA2 database [8] to search for the expression level of WDR62 in all cancer datasets. The ILog ${ }_{2} \mathrm{FCI}$ cutoff is 1 . $P$ value cutoff is 0.05 . Jitter size is 0.4 . Matched normal data contains TCGA normal and GTEx data. Besides, we obtained the WDR62 expression data in different stages via the "Stage Plot" module. We used the "Survival Map" module to acquire the overall survival (OS) and disease-free survival (DFS) map data of WDR62 across all tumors. The group cutoff is median by cutoff high (50\%) and cutoff low (50\%). Significance level is 0.05 . Then, the "Survival Analysis" module was used to analyze the detailed OS and DFS data of single cancer. We obtained the hazard ratio (HR) and $P$ value by the log-rank test. We used the "Correlation Analysis" module to analyze the correlation between WDR62 expression and CD274 (also known as PD-L1) expression in tumor tissues by Spearman correlation coefficient. $P$ value cutoff is 0.05 .

2.2. TIMER Database Analysis. We used the "Diff Exp" module of the TIMER database $[9,10]$ to dissect the expression level of WDR62 in different tumors. Statistical significance of different expression was evaluated using the Wilcoxon test. $P$ value cutoff is 0.05 . The "Survival" module allows us to compare the overall survival between high and low WDR62 expression level groups, under the setting of cutoff high (50\%) and cutoff low (50\%). The "Correlation" module was used to analyze the correlation between WDR62 expression and CD274 expression in tumor tissues by Spearman correlation coefficient. $P$ value cutoff is 0.05 . And we used the "Gene" module to analyze the association between WDR62 expression and immune cell infiltration. Immune cells contain B cell, CD4+ T cell, CD8+ T cell, macrophage, neutrophil, and dendritic cell. $P$ value cutoff is 0.05 . Subse- quently, we continued to analyze the correlation between WDR62 expression and cancer-associated fibroblasts (CAF) and $\mathrm{T}$ cell regulatory (Treg) cells in the "Gene" module of TIMER2 (Tumor Immune Evaluation Resource, version 2). And we explored the overall survival in a multivariable Cox hazard model. Covariates are WDR62 expression and CAF or Treg infiltration levels. Increased risk was set as $P$ value $<0.05$ and $Z$ score $>0$. The data was visualized as a heat map and a scatter plot. Moreover, we used the "Gene-Corr" module to draw the heat map of relative genes in different tumors. $P$ value cutoff is 0.05 .

2.3. UALCAN Database Analysis. The mRNA expression of WDR62 in tumor and normal tissues was determined within the UALCAN database [11]. And we compared WDR62 expression in breast cancer among different stages, races, ages, subclasses, histologic subtypes, nodal metastasis status, and TP53 mutation status. Moreover, the overall survival data and promoter methylation status of WDR62 in candidate tumors were analyzed. $P$ value cutoff is 0.05 .

2.4. StarBase Database Analysis. The expression of WDR62 in tumor and normal tissues was determined within the StarBase V3.0 database [12]. And the overall survival data of WDR62 in different cancers was analyzed. Moreover, the correlation between WDR62 expression and CD274 expression in tumor tissues was explored. $P$ value cutoff is 0.05 .

2.5. TISIDB Database Analysis. The TISIDB database was used to analyze the association between WDR62 expression and overall survival and stage data across the candidate tumors [13]. Furthermore, we compared the correlation between WDR62 expression and immunoinhibitors. And the correlation between WDR62 expression and CD274 or CTLA4 expression in tumor tissues was analyzed by Spearman correlation coefficient. $P<0.05$ was considered statistically significant. Finally, we compared the WDR62 expression difference between responders and nonresponders receiving PD1/PD-L1 inhibitors. $P<0.05$ was considered statistically significant.

2.6. TCGA Portal Database Analysis. The correlation between WDR62 expression and prognostic significance was analyzed by specialized prognostic database-TCGA portal [14]. The overall survival (OS) data was obtained based on TCGA datasets. $P$ value $<0.05$ was considered statistically significant.

2.7. Kaplan-Meier Plotter Database Analysis. Kaplan-Meier Plotter is able to assess the survival data based on GEO, EGA, and TCGA datasets, promoting the discovery and validation of survival biomarkers [15]. The prognostic value of WDR62 expression including the overall survival (OS), disease-free survival (DFS), first progression survival (FP), and relapse survival (PPS) was evaluated. Furthermore, the WDR62 prognostic value along with immune cell infiltration including CD4+ T cell, CD8+ T cell, macrophage, natural killer (NK) T cells, and regulatory $\mathrm{T}$ cells (Treg) was compared. $P$ value $<0.05$ was considered statistically significant. 


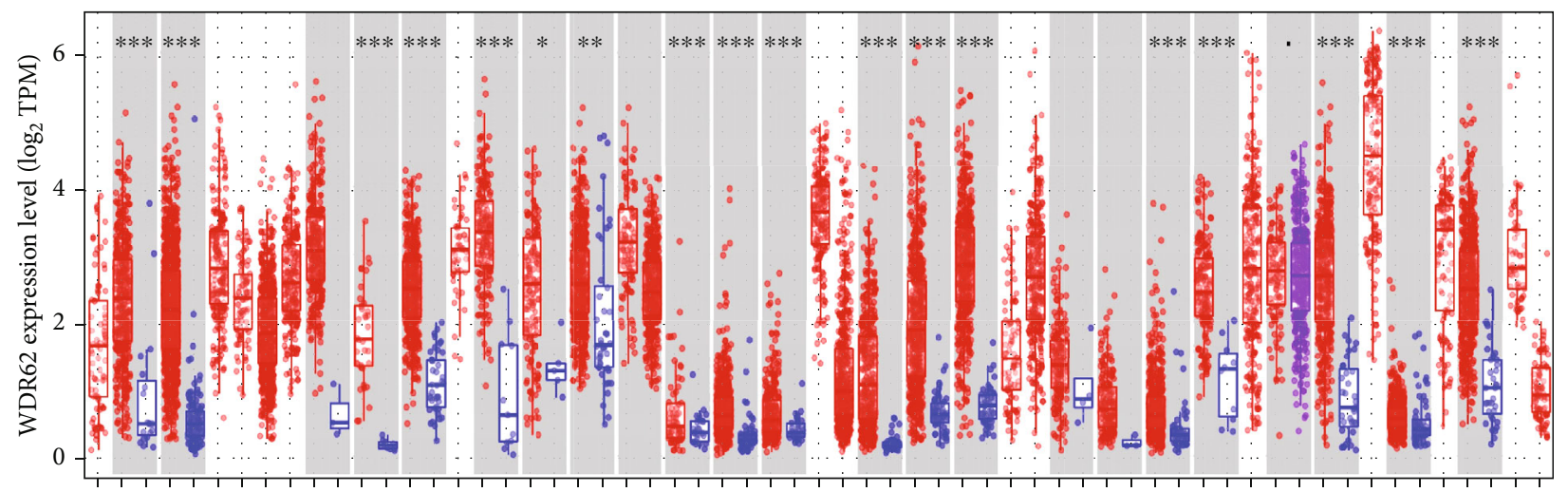

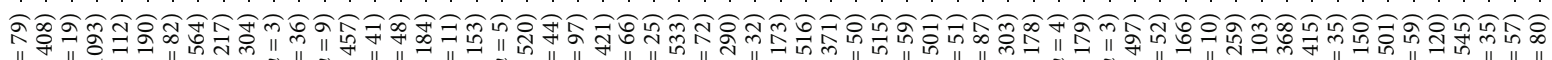

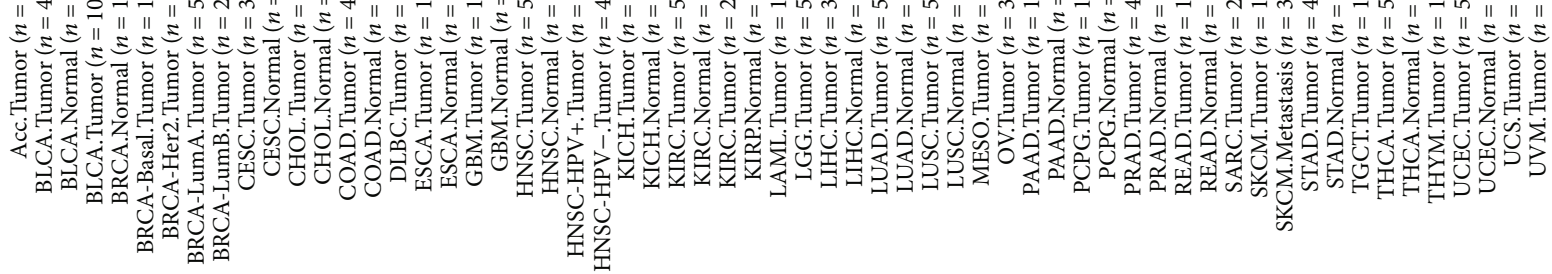

(a)
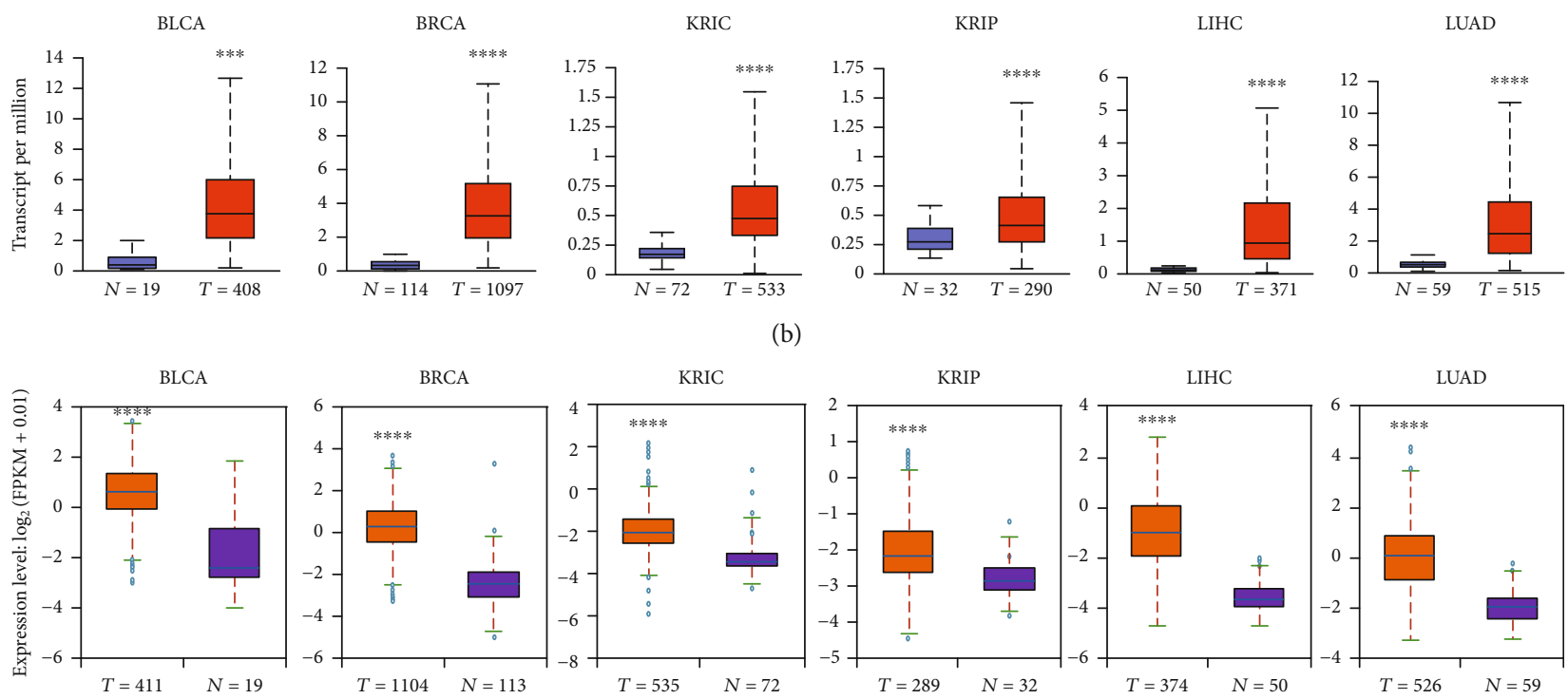

(c)

FIGURE 1: Expression level of WDR62 in different tumors. (a) The expression level of WDR62 in different tumors or specific tumor subtypes was analyzed through the TIMER database. (b) The expression level of WDR62 between normal tissue and primary tissue of BLCA, BRCA, KIRC, KIRP, LIHC, and LUAD in the UALCAN database. (c) The expression level of WDR62 between normal tissue and primary tissue of BLCA, BRCA, KIRC, KIRP, LIHC, and LUAD in the StarBase database.

2.8. PrognoScan Database Analysis. The correlation between WDR62 expression and prognostic significance was analyzed by specialized prognostic database-PrognoScan [16]. The overall survival (OS), disease-specific survival (DSS), and disease-free survival (DFS) data were obtained based on the GEO datasets. $P$ value $<0.05$ was considered statistically significant.

2.9. STRING Analysis. The protein-protein interaction (PPI) networks of WDR62 were analyzed by online tool STRING
[17]. We set the parameters as follows: network type (full STRING network), meaning of network edges (evidence), active interaction sources (experiments), minimum required interaction score (low confidence (0.150)), and max number of interactors to show (no more than 50 interactors).

2.10. GeneMANIA Database Analysis. The GeneMANIA database was used for exploring the interaction network for WDR62 [18, 19]. The bioinformatic methods involved gene physical interaction, coexpression, prediction, colocation, 

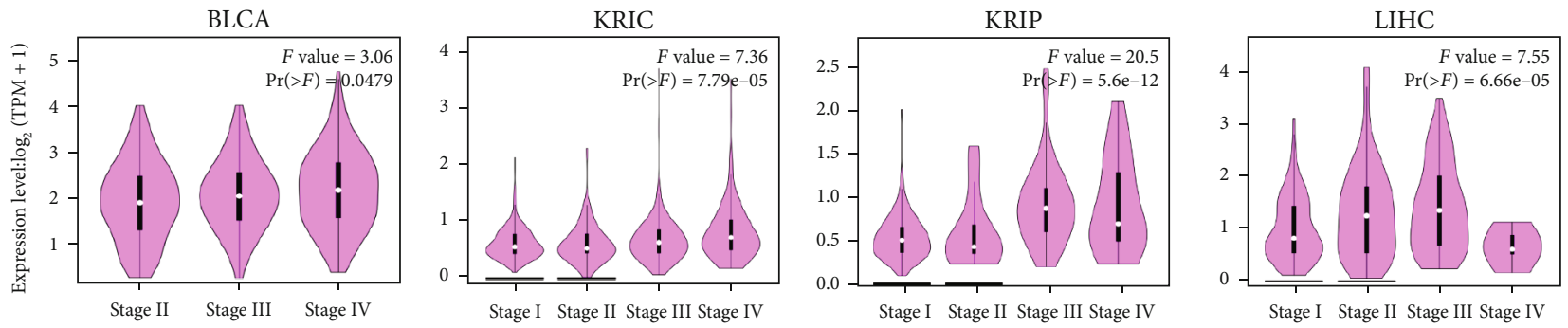

(a)

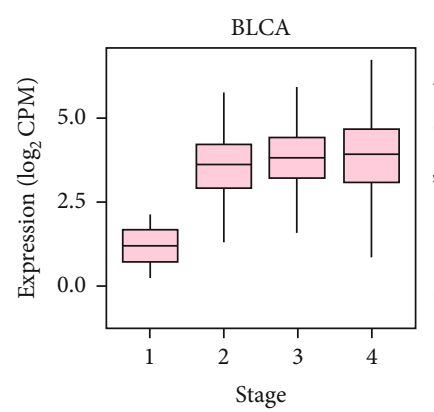

Spearman: $\mathrm{rho}=0.119, p=0.016$

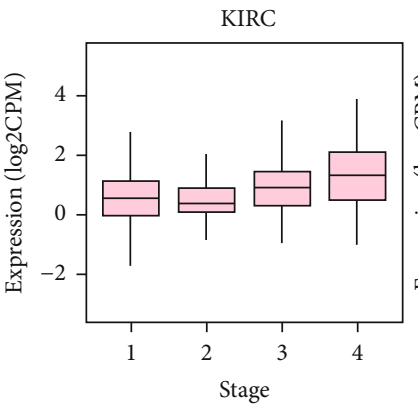

Spearman: rho $=0.233, p=5.27 \mathrm{e}-08$

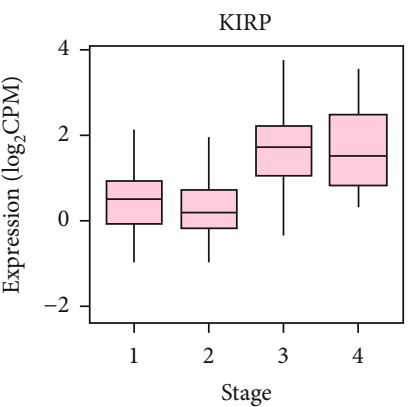

Spearman: rho $=0.411, p=5.26 \mathrm{e}-12$

(b)

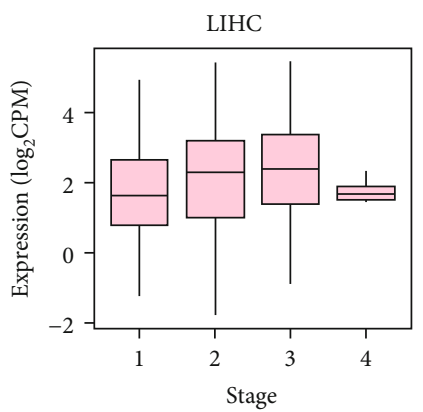

Spearman: rho $=0.176, p=0.00102$

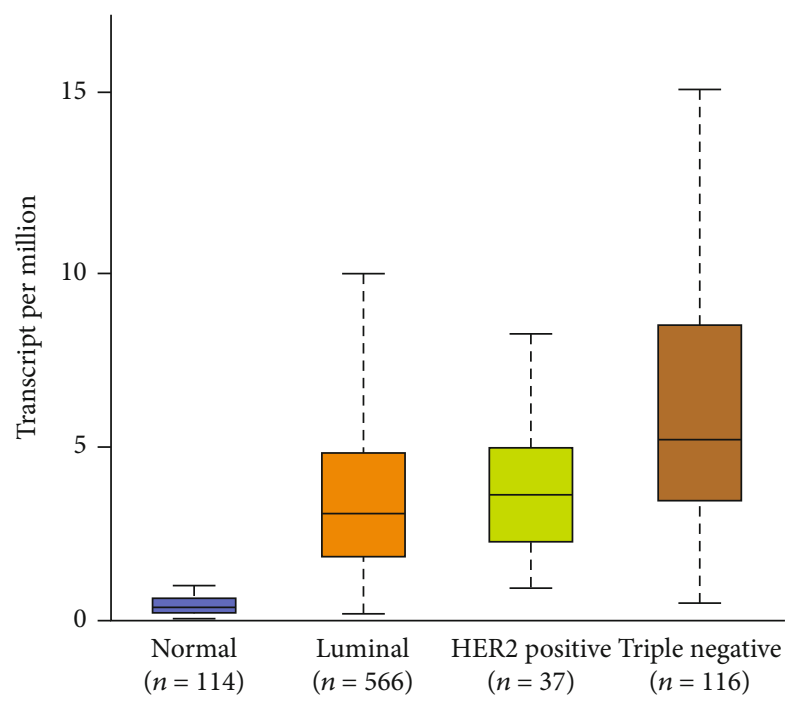

(c)

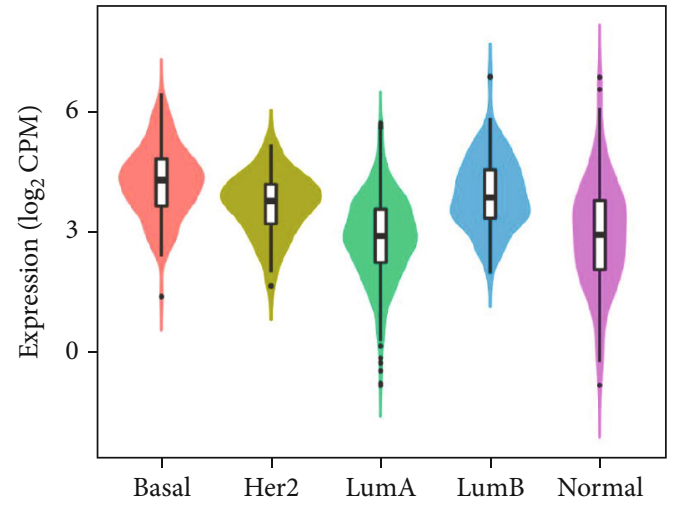

(d)

Figure 2: Continued. 


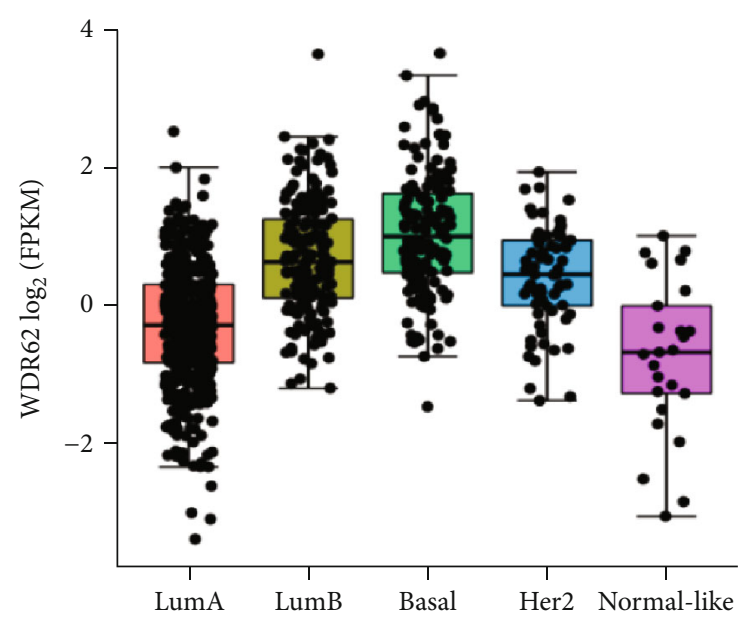

(e)

FIGURE 2: Expression level of WDR62 in different pathological stages and subtypes. (a, b) Based on TCGA data in the GEPIA2 and TISIDB database, the expression levels of WDR62 were analyzed in the main pathological stages of BLCA, KIRC, KIRP, and LIHC. (c-e) The expression level of WDR62 in different subtypes of BRCA in UALCAN, TISIDB, and TCGA portal databases.

pathway, genetic interaction, and shared protein domains. We visualized the top 20 gene networks through the GeneMANIA.

2.11. DAVID Analysis. The interactive genes of WDR62, conducted by STRING and GeneMANIA databases, were all input into Database for Annotation, Visualization, and Integrated Discovery (DAVID) online tool for Gene Ontology (GO) and related pathway analysis [20]. GO analysis contains biological process (BP), molecular function (MF), and cellular component (CC). Related pathways contain Kyoto Encyclopedia of Genes and Genomes (KEGG) pathways and Reactome pathways. $P$ value $<0.05$ was considered statistically significant.

2.12. Statistics. Student's $t$-test was performed for statistical significance analysis. $P$ value $<0.05$ was considered as statistically significant; ${ }^{*} P<0.05,{ }^{* *} P<0.01,{ }^{* * *} P<0.001$, and *** $P<0.0001$.

\section{Results}

3.1. Gene Expression Analysis Data. Given the heterogeneity of tumor, single TCGA dataset could not reflect accurately the real expression level of genes. We firstly analyzed the expression level of WDR62 in 4 different databases-GEPI A2, TIMER, UALCAN, and StarBase. We discovered that WDR62 was overexpressed in over 20 tumor types (Figure 1(a) and S-Figure 1). The prognostic value was analyzed in 8 different databases-GEPIA2, TIMER, UALCAN, StarBase, TISIDB, TCGA portal, Kaplan-Meier Plotter, and PrognoScan. We selected 6 candidate tumors-bladder urothelial carcinoma (BLCA), breast invasive carcinoma (BRCA), kidney renal clear cell carcinoma (KIRC), kidney renal papillary cell carcinoma (KIRP), liver hepatocellular carcinoma (LIHC), and lung adenocarcinoma (LUAD), since WDR62 was overexpressed in at least 3 different databases and also had potential prognostic value in at least 2 databases among these tumors (Figures 1(b) and 1(c)). Moreover, we observed a correlation between WDR62 expression and the pathological stages of tumors, contributing to the diagnosis of tumor stage (Figures 2(a) and 2(b)). Notably, among different subtypes of BRCA, the expression level of WDR62 in triple-negative breast cancer (TNBC) was higher than other types including Her2, LumA, and LumB type (Figures 2(c) $-2(e)$ ), implying the possibility that WDR62 participates in the tumorigenesis of TNBC. Additionally, WDR62 expression level was associated with tumor stage, subclass, histologic subtype, patient's age, and race in BRCA (S-Figure 2). In summary, WDR62 could serve as a diagnostic biomarker in most of the tumors.

3.2. Survival Analysis Data. Subsequently, we investigated the prognosis value of WDR62 in different tumors. By GEPIA2 database, we performed the survival map of WDR62 across all tumors and analyzed the OS and DFS in detail in 6 candidate tumors (Figure 3(a)). Interestingly, high-expressed WDR62 indicated poor prognosis including OS and DFS of KIRC, KIRP, LIHC, and LUAD in databases based on TCGA datasets (Figure 3(b), S-Figure 3A-C). But there was no prognostic value in BLCA and BRCA even with higher WDR62 expression in these databases. However, it is notable that high-expressed WDR62 in basal-type BRCA implied poor OS in the TIMER database, suggesting different roles of WDR62 in BRCA subtypes (S-Figure 3B). We continued to analyze the survival data of WDR62 using Kaplan-Meier Plotter and PrognoScan databases based on GEO datasets. Unexpectedly, we identified a correlation between WDR62 expression and poor prognosis in BLCA and BRCA (Figure 3(c) and S-Figure 3D). Consequently, the above data indicated that WDR62 could serve as a prognostic biomarker in specific tumor types.

3.3. Driver Gene and Promoter Methylation Analysis Data. Now that WDR62 is overexpressed in most of the tumors, we are curious about the reasons. By TCGA portal database, 


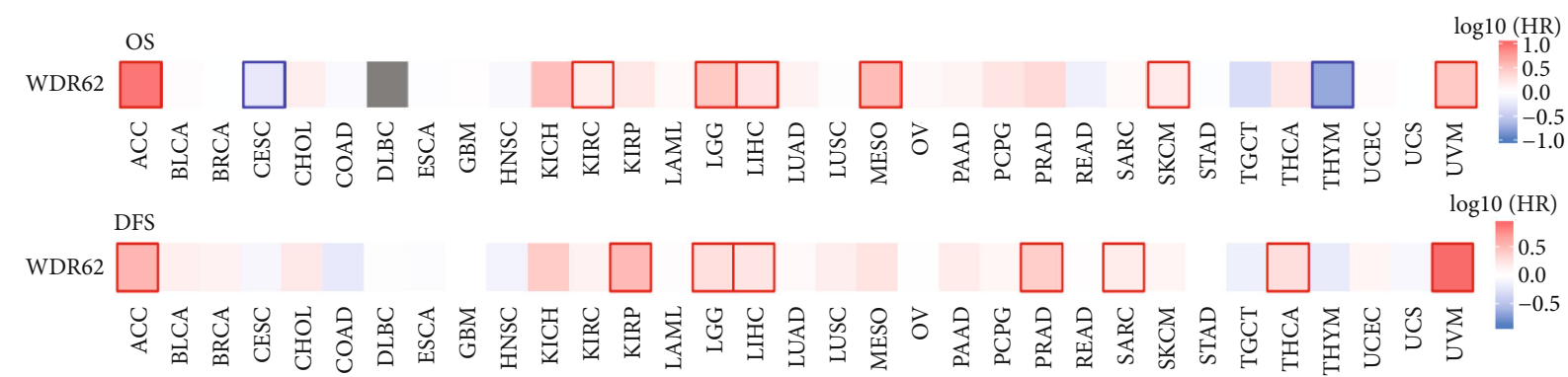

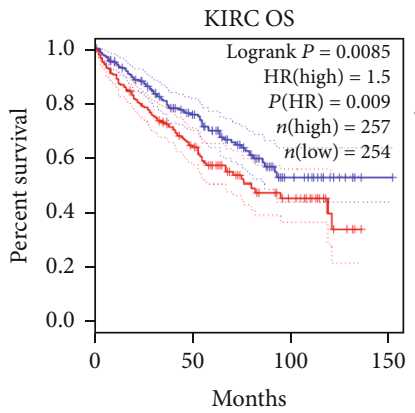

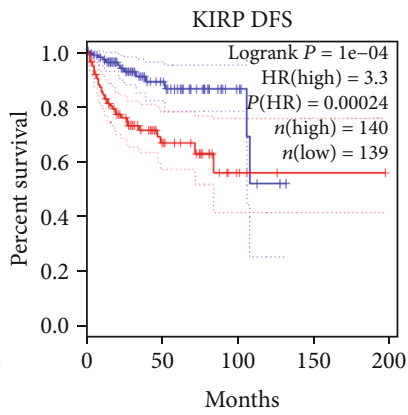

— Low WDR62 TPM

— High WDR62 TPM
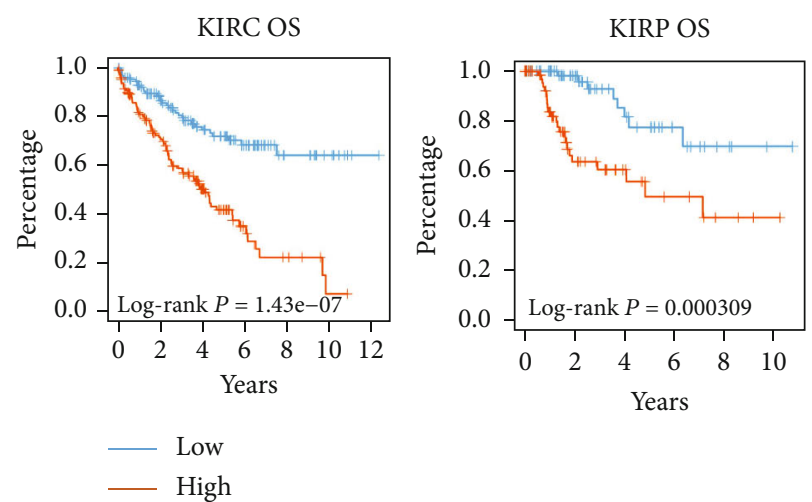
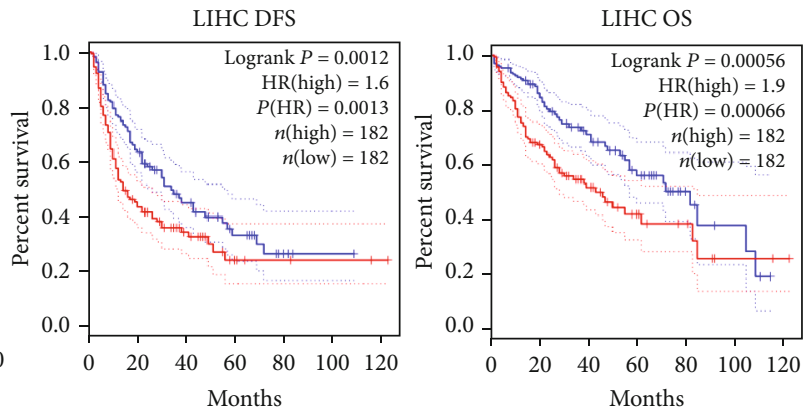

(a)
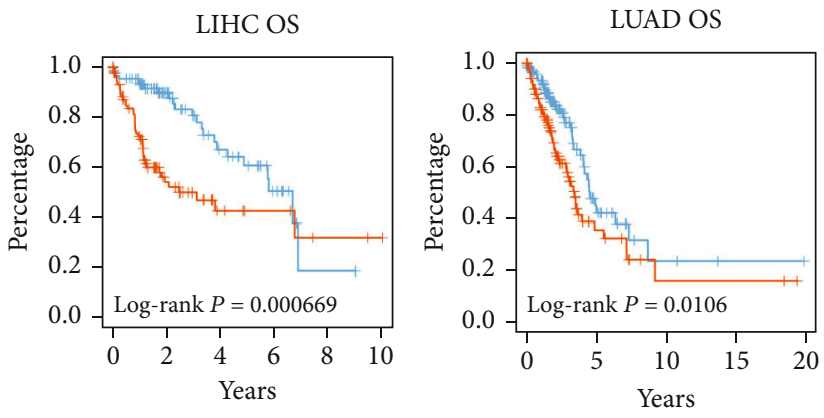

(b)

Figure 3: Continued. 

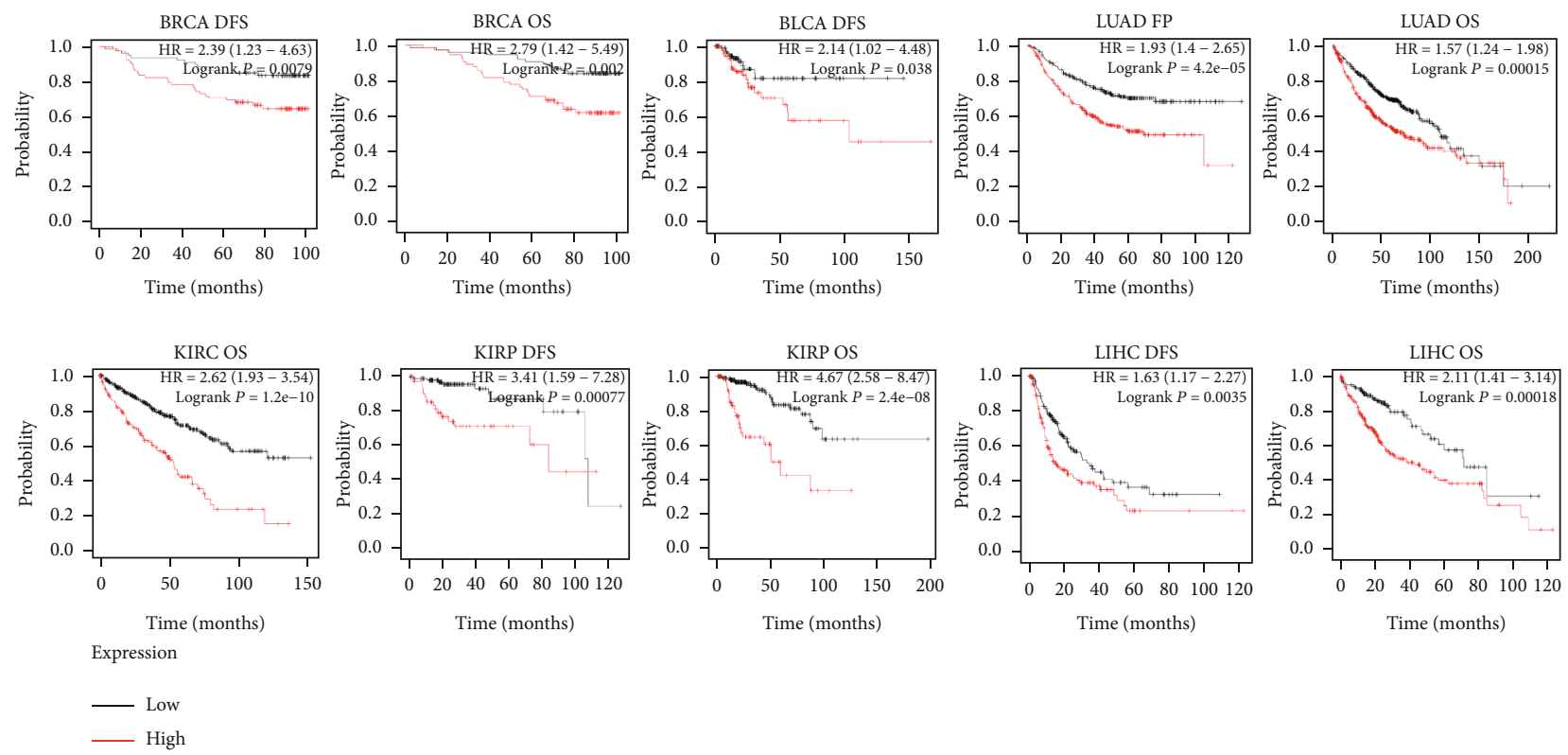

(c)

FIGURE 3: Correlation between WDR62 expression and survival prognosis of tumors. (a) The survival map of WDR62 was analyzed by GEPI A2. (b) Prognosis value-OS of KIRC, KIRP, LIHC, and LUAD in the TISIDB database. (c) The survival value of WDR62 using the KaplanMeier Plotter.

we compared the correlation between WDR62 expression and important driver genes in BRCA, KIRC, LIHC, and LUAD, including PIK3CA, TP53, PTEN, BRCA1, MTOR, CTNNB1, KRAS, EGFR, and BRAF. As shown in Figure 4(a), TP53 showed the highest correlation in all these 4 tumors, providing a cause for WDR62 overexpression. And we confirmed that WDR62 expression was significantly associated with mutated TP53 in the UALCAN database, further validating the result (Figure 4(b)). Meanwhile, we observed an obvious negative correlation between WDR62 expression and its promoter DNA methylation (Figure 4(c)), suggesting another possibility.

3.4. Enrichment Analysis of WDR62-Related Genes. To further explore the molecular mechanism of WDR62 in carcinogenesis, we screened out WDR62-binding proteins by STRING online tool. We acquired a total of 27 genes supported by experimental evidence (Figure 5(a)). Subsequently, we investigated the interaction network for WDR62 by the GeneMANIA database. Figure 5(b) shows the top 20 genes and their relationship with WDR62. We compared the two sets of genes and discovered 4 common members-CEP170, MAPK8, MAPK9, and MAPK10 (Figure 5(c)). Then, we used the GEPIA2 database to obtain the correlation heat map data in all tumor types (Figure 5(d)). As shown in Figure 5(e), WDR62 expression was positively correlated with that of CEP170, MAPK8, and MAPK9 in the majority of tumors. In contrast, WDR62 expression was negatively correlated with that of MAPK10 in the majority of tumors. Finally, we combined the two groups of genes and performed GO enrichment and KEGG pathway analysis via DAVID online tool (S-Figure 4A-D). Results demonstrated that WDR62 relative genes may be mainly involved in the MAPK signaling pathway (S-Figure 4E).

3.5. Immune Infiltration Analysis Data. Other than the MAPK pathway, WDR62 was also closely correlated with the toll-like receptor signaling pathway, which is involved in immune reaction. Therefore, we are keen to explore the association between WDR62 expression and immune features. Firstly, we analyzed a series of immune cells via the TIMER database in 6 candidate tumors. The results showed that WDR62 expression was closely correlated with the infiltration level of several immune cells, including B cell, CD4+ T cell, CD8+ T cell, macrophage, neutrophil, and dendritic cell (S-Figure 5). More importantly, WDR62 expression was associated with the abundance of CAF and Treg cells by using TIMER2 platform analysis (Figures 6 and 7). Besides, we observed that the prognosis of patients with high WDR62 expression plus high CAF or Treg is the worst in KIRP and KIRC, respectively (Figure 8). GEO datasets also indicated that high WDR62 expression predicted worse prognosis with high Treg cell infiltration or low immune cell infiltration such as CD4+ T cell, CD8+ T cell, macrophage, and NK cell (S-Figure 6).

3.6. Association Analysis with Immunomodulators. With the popularity of immunotherapy, in particular PD1/PD-L1 inhibitors, we wondered whether WDR62 was relevant with immunomodulators. By coincidence, in 3 databases, the expression of WDR62 was positively correlated with PD-L1 (CD274) expression in 5 candidate tumors except KIRP (Figures 9(a) - 9(c)). Notably, WDR62 expression in triplenegative breast cancer (TNBC) was more correlated with PD-L1 expression than Her2 and luminal subtype 
BRCA

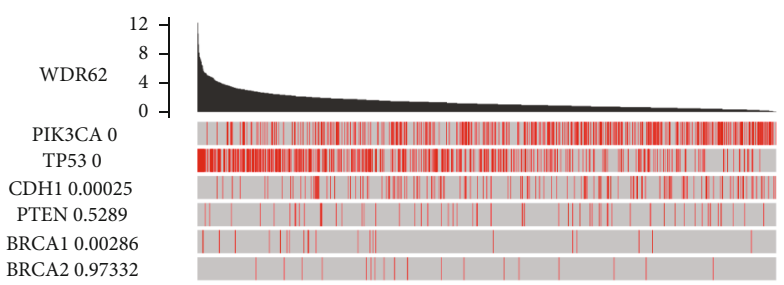

LIHC

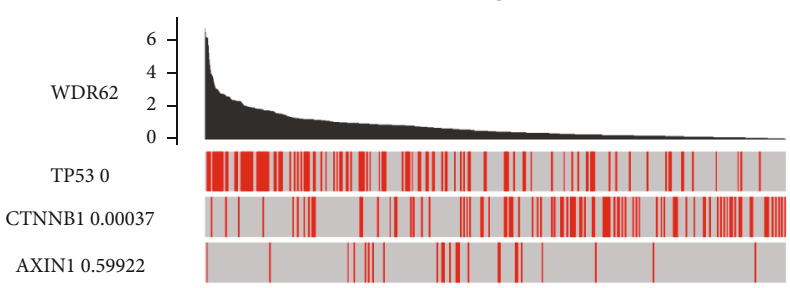

KIRC

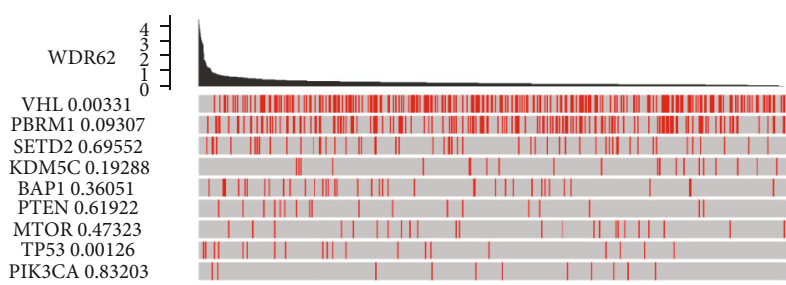

LUAD

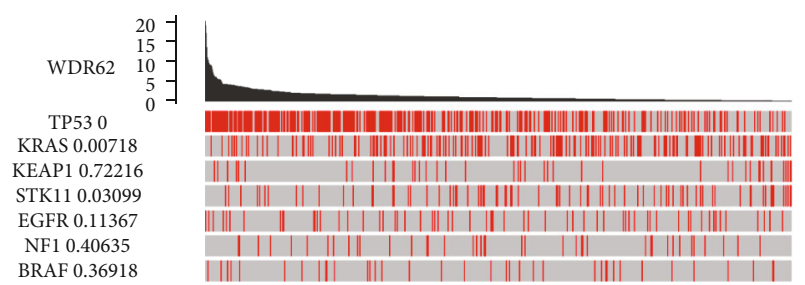

(a)

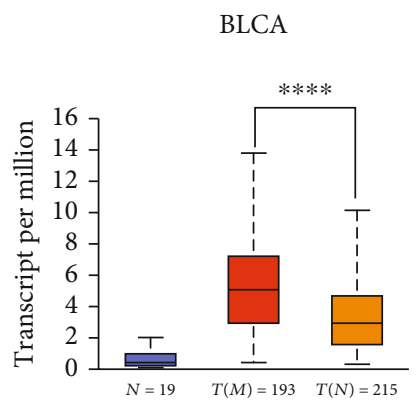

BRCA
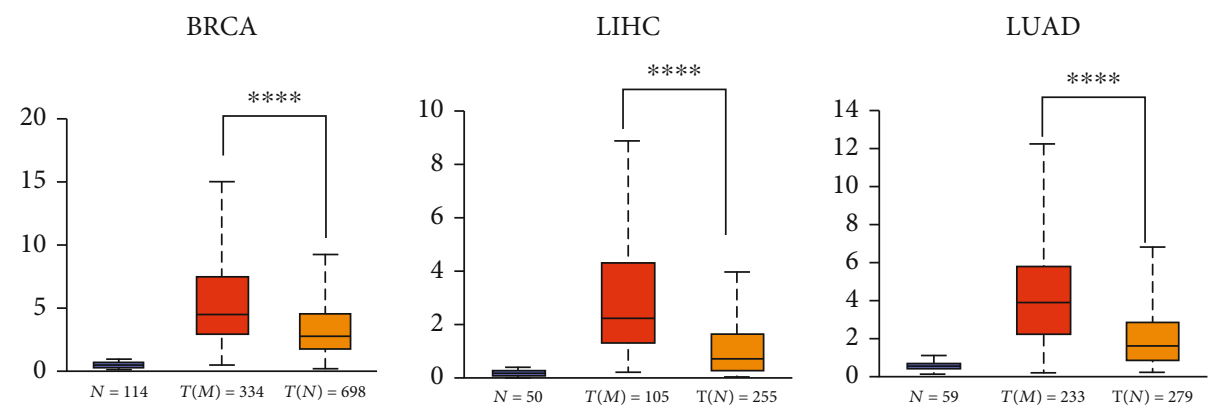

(b)
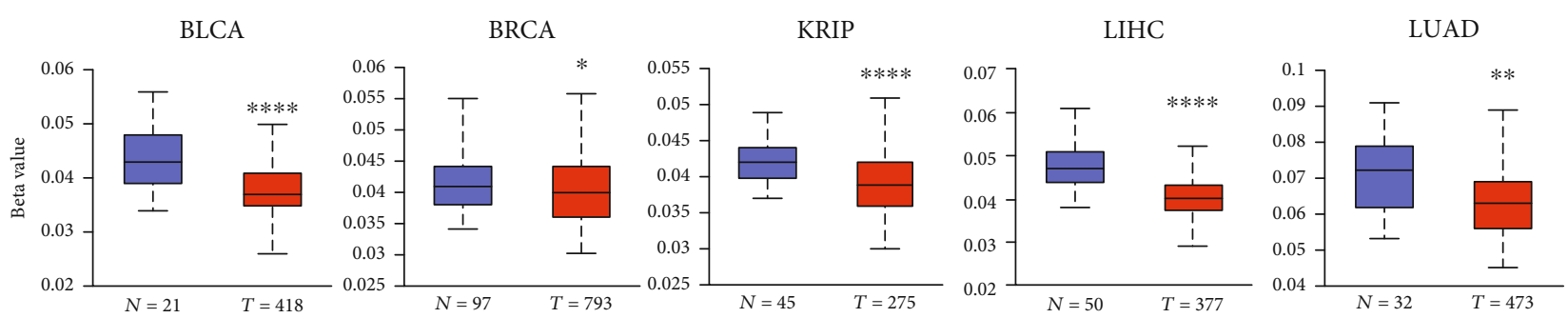

(c)

FIGURE 4: Correlation between WDR62 expression and driver gene and promoter methylation. (a) The value adjacent to highly mutated gene is permutation test $P$ value of gene expression between driver mutated (red) and not mutated (gray) samples. (b) Correlation between WDR62 expression and mutated TP53 in the UALCAN database. (c) Correlation between WDR62 expression and promoter DNA methylation in the UALCAN database.

(Figure 9(d)), in accordance with the above data about WDR62's role in TNBC. To further investigate the relationship between WDR62 and immunomodulators, we performed Spearman's correlation analysis via the TISIDB database. We found that WDR62 expression was mostly positively correlated with the abundance of immunoinhibitors such as PD-L1 and CTLA4 (Figures 10(a) and 10(b)). Finally, we discovered that WDR62 had significant difference of expression between responders and nonresponders receiving atezolizumab (anti-PD-L1) in urothelial cancer (Figure 10(c)). Collectively, these results demonstrated that WDR62 may indeed be involved in tumor immune regulation.

\section{Discussion}

Despite the great advances in diagnosis and treatment of tumors, it is still threatening human survival. Hence, there is an urgent need to identify new tumor biomarkers beneficial for early diagnosis of tumor and the prediction of cancer therapeutic effect. It has been reported that WDR62 plays crucial roles in many cellular processes such as spindle maintenance, cell cycle progression, and cell proliferation [21-23]. Recently, accumulating evidence suggests that WDR62 is a new identified tumor biomarker in few tumors such as lung cancer and bladder cancer $[24,25]$. Shinmura et al. suggested WDR62 was overexpressed in LUAD and was associated 


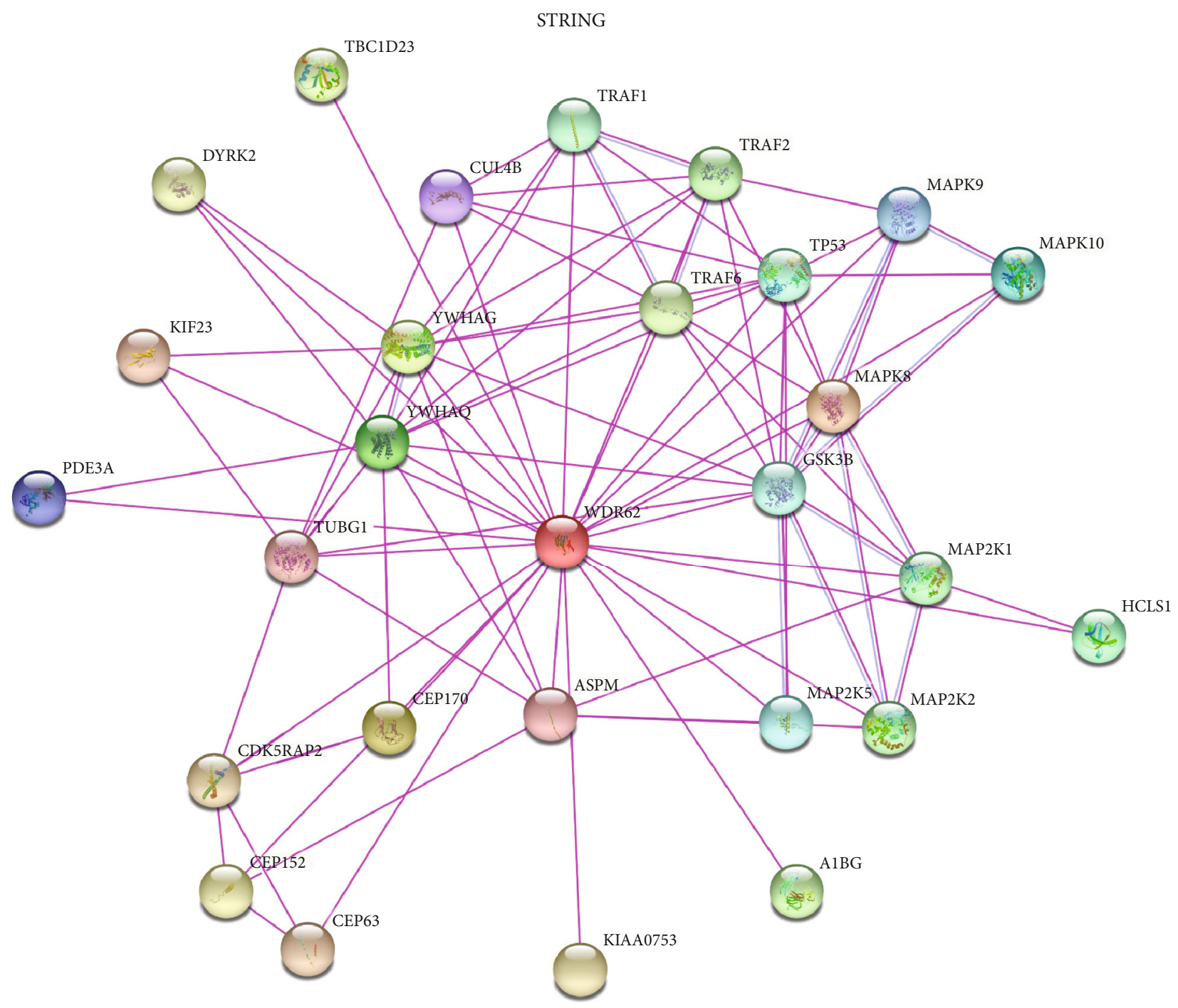

(a)

Figure 5: Continued. 


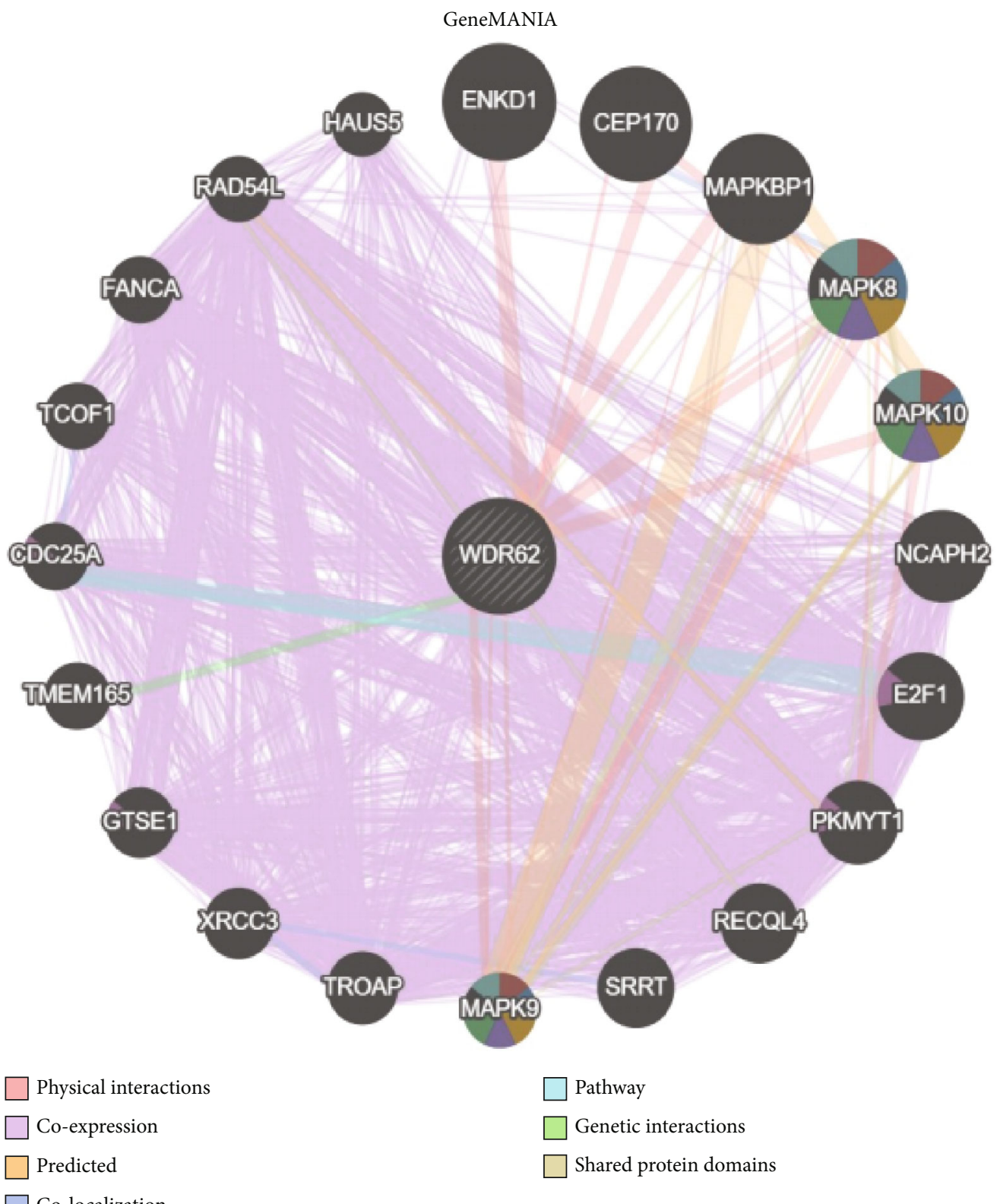

Co-localization

(b)

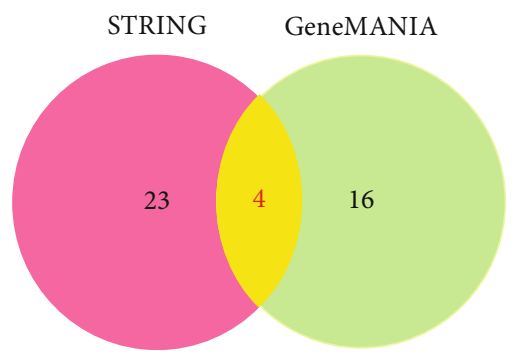

(c)

FIgURe 5: Continued. 


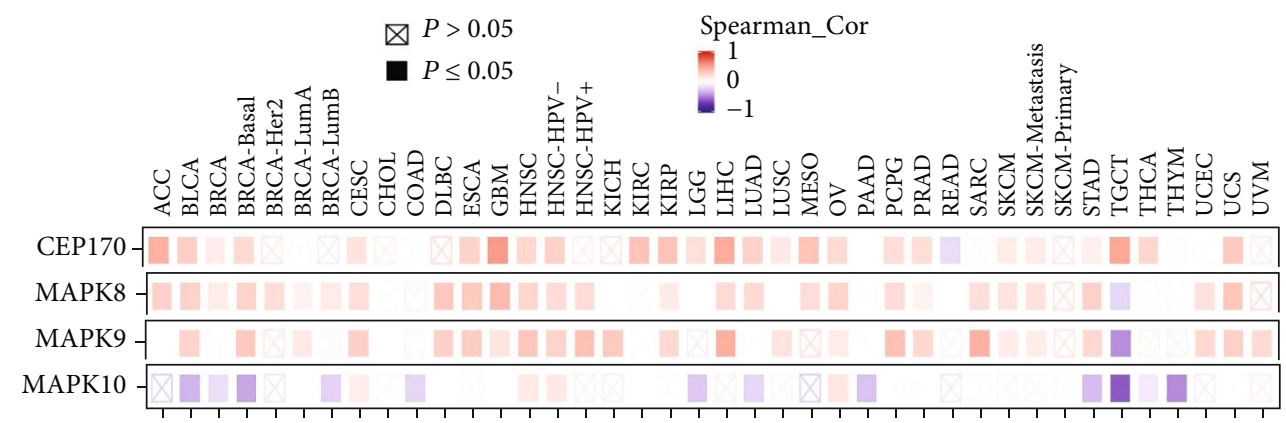

(d)
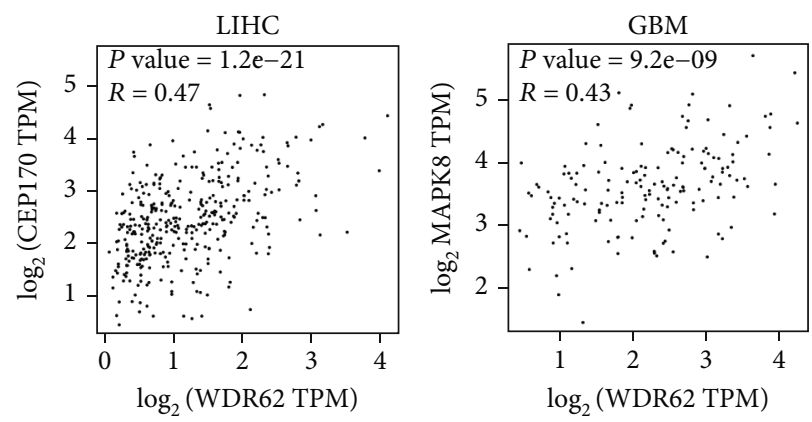
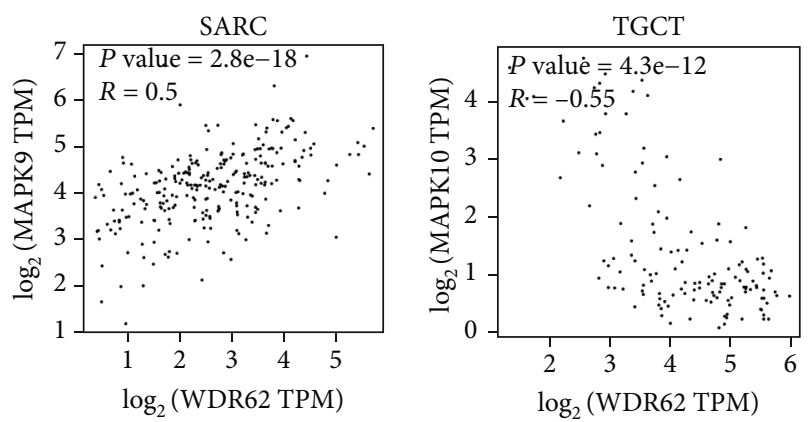

(e)

FIGURE 5: WDR62-related gene enrichment analysis. (a) The available experimentally determined WDR62-binding proteins using the STRING tool. (b) Using the GeneMANIA approach, top 20 WDR62-correlated genes were analyzed. (c) An intersection analysis of the WDR62-binding and correlated genes was conducted. (d) The corresponding heat map data in the detailed cancer types is displayed. (e) GEPIA2 was used to analyze the correlation between WDR62 and selected targeting genes, including CEP170, MAPK8, MAPK9, and MAPK10.

with a poor prognosis. In order to figure out the oncogenic role of WDR62, we performed a pan-cancer analysis from the view of overall tumors based on bioinformatic data.

In this study, we identified that WDR62 could serve as a diagnostic biomarker in most of the tumors since WDR62 was overexpressed simultaneously in at least 3 different databases. In breast cancer, with the highest morbidity now, we further analyzed the datasets and discovered a correlation between WDR62 expression and clinical features. More importantly, we observed that WDR62 expression in triplenegative breast cancer (TNBC) was higher than that in other subtypes, suggesting that a distinct mechanism may exist for WDR62 in BRCA subtypes. Subsequently, our research indicated that WDR62 could also serve as a prognostic biomarker mainly in 6 tumor types. Among them, KIRC, KIRP, and LIHC showed the best prognostic value due to its significance in most databases. Though BLCA and BRCA did not have prognostic value via TCGA-based databases, we found a survival difference in GEO-based databases, suggesting that there was a discrepancy about prognostic value in different databases based on TCGA or GEO datasets. Extraordinarily, our study demonstrated that high-expressed WDR62 implied poor survival in TNBC, further highlighting its potential as a biomarker in TNBC.

The research on the molecular mechanism and functional enrichment analysis of WDR62 in tumors is seldom. For instance, Sugita et al.'s study reported that microRNA223 was responsible for the abnormal expression of WDR62 in bladder cancer [25]. Therefore, we first applied
TCGA datasets to explore the molecular mechanism for WDR62 high expression. Our findings demonstrated highexpressed WDR62 was subject to multiple driver genes in particular TP53 which showed the highest correlation in all candidate tumors. It is of interest to further explore the mechanism between WDR62 and TP53 in future study. Besides, we discovered a correlation between WDR62 expression and its promoter DNA methylation, providing another cause for WDR62 high expression. Subsequently, we integrated the information about WDR62-binding proteins and WDR62-relevant genes for enrichment analysis and identified the potential effect on the MAPK and toll-like receptor signaling pathway, which was consistent with previous findings that JNK, one MAPK cascade, was activated by WDR62 in tumor cells [26]. More importantly, we are the first study to present evidence about the correlation between WDR62 expression and immune cell infiltration in 6 candidate tumors. Extraordinarily, a significant positive correlation was suggested between WDR62 expression and the abundance of CAF and Treg cells. And the patients with high WDR62 expression plus high CAF or Treg cell infiltration have the worst overall survival. The prominent components of tumor microenvironment are immune cells which were related with tumorigenesis, progression, and metastasis [27, 28]. Accordingly, we considered that WDR62 may play vital roles in tumorigenesis by regulating the tumor microenvironment (TM). By coincidence, we found a significant expression of exosomal WDR62 in colorectal cancer, hepatocellular carcinoma, and pancreatic adenocarcinoma by the 


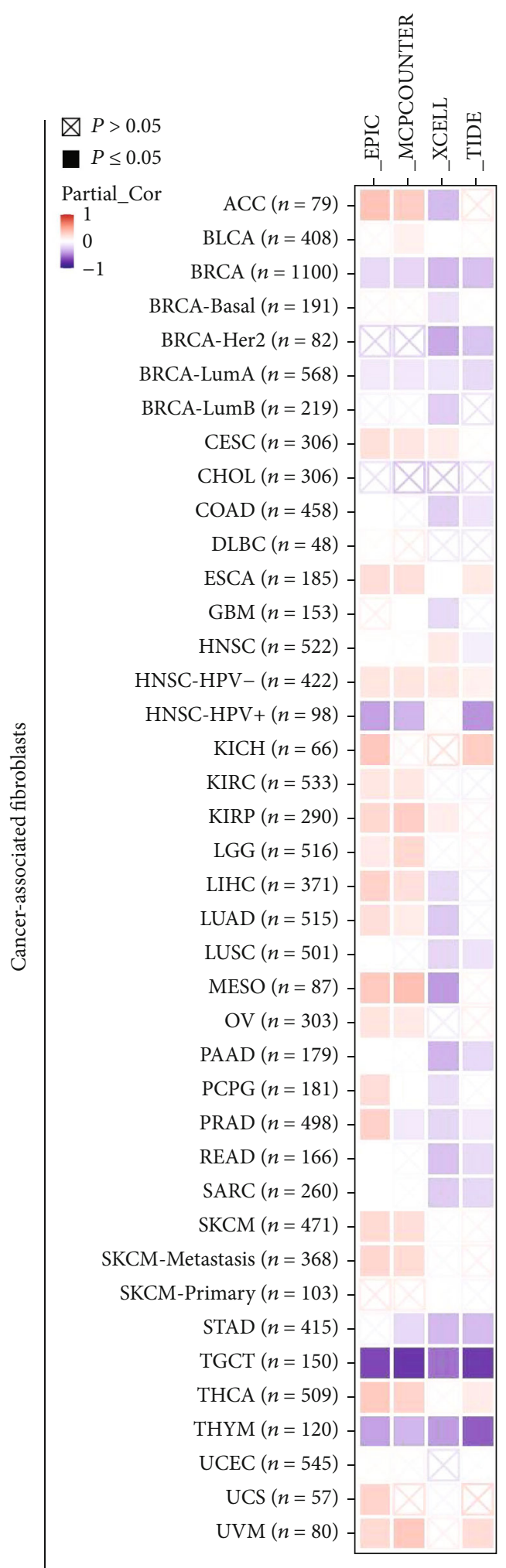

(a)

Figure 6: Continued. 

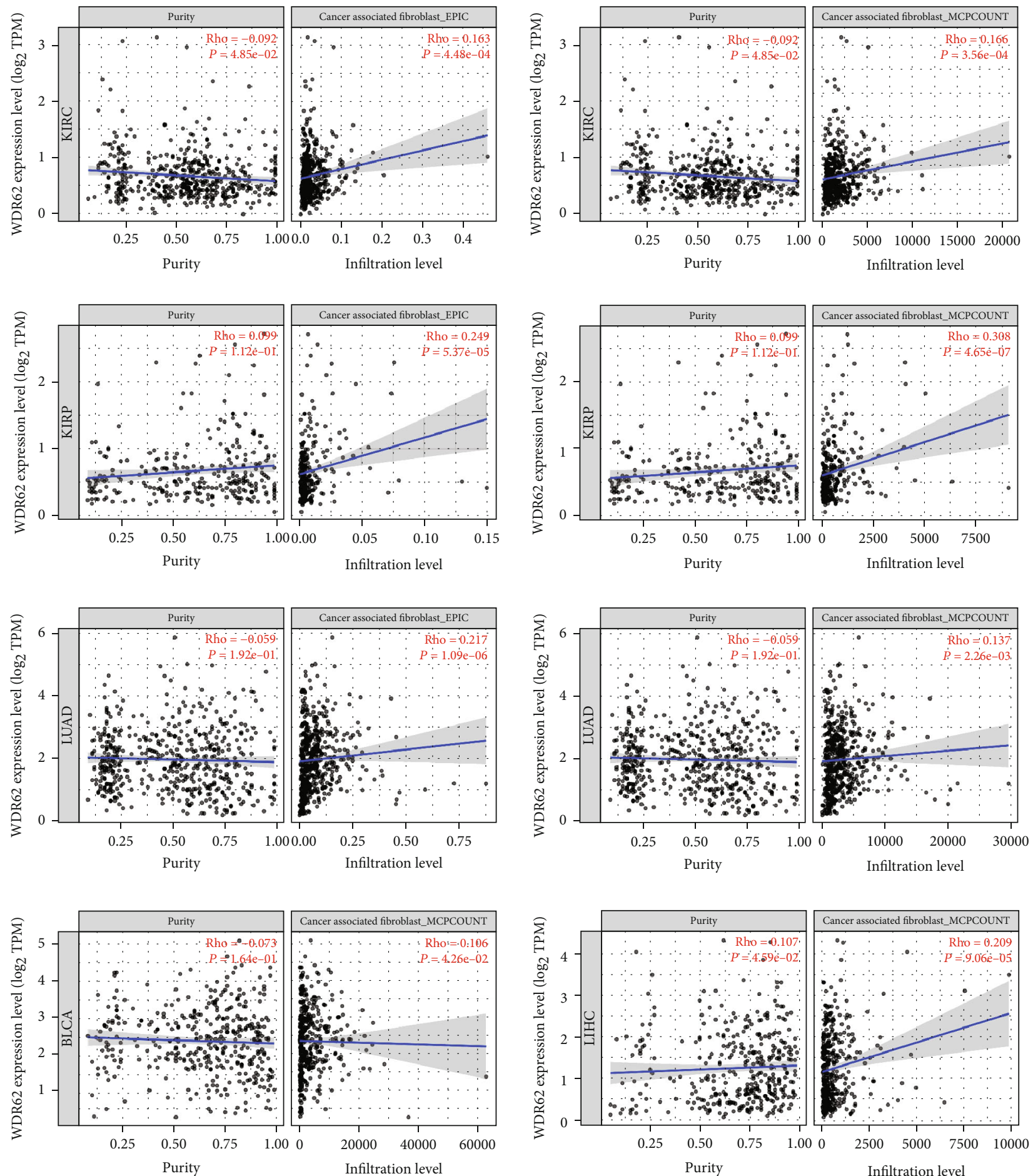

(b)

FiguRE 6: Correlation analysis between WDR62 expression and immune infiltration of CAF. (a) The heat map about correlation between WDR62 expression and cancer-associated fibroblasts (CAF). (b) The correlation between WDR62 expression and cancer-associated fibroblasts (CAF) in BLCA, KIRC, KIRP, LIHC, and LUAD.

exoRBase database (S-Figure 4F). Given that exosomes exhibit their property in intercellular communication between tumor cells and tumor microenvironment [29], we inferred that the regulatory effect on CAF or Treg cells may be by means of exosomal WDR62.
Immunotherapy in particular PD1/PD-L1 inhibitors has aroused great interest in recent years. Detecting PD1/PD-L1 expression levels by immunohistochemistry was treated as standard assay for applying PD1/PD-L1 inhibitors [30]. However, there are significant differences in responsiveness 


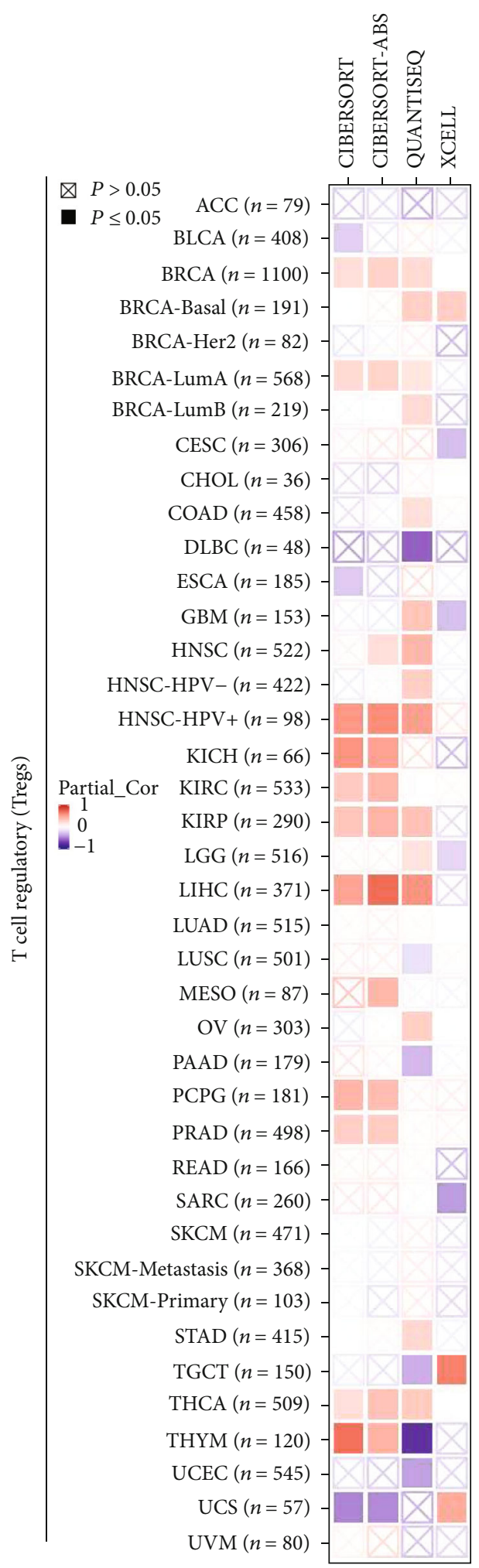

(a)

Figure 7: Continued. 

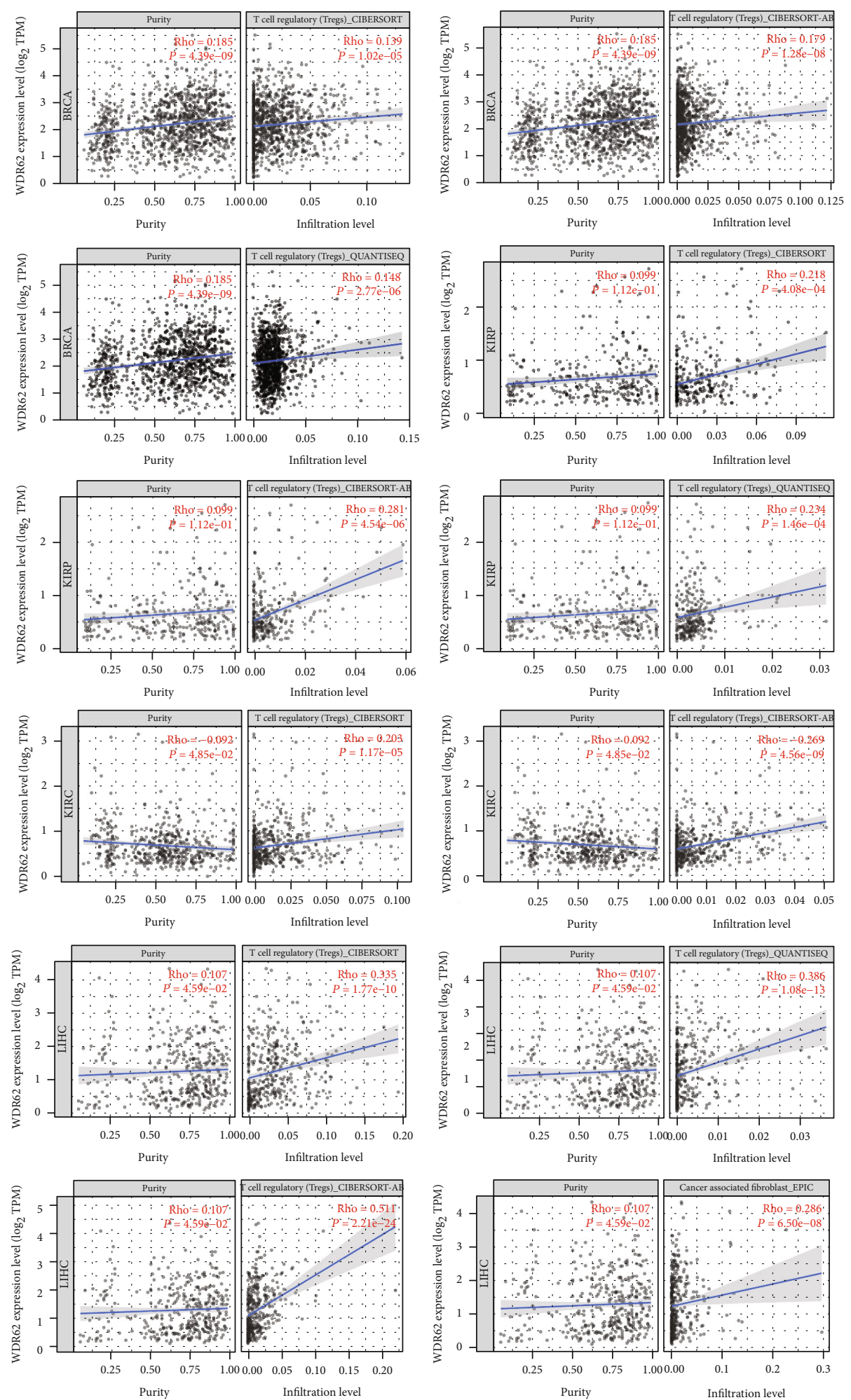

(b)

FIGURE 7: Correlation analysis between WDR62 expression and immune infiltration of Treg cells. (a) The heat map about correlation between WDR62 expression and Treg cells. (b) The correlation between WDR62 expression and Treg cells in BRCA, KIRC, KIRP, and LIHC. 


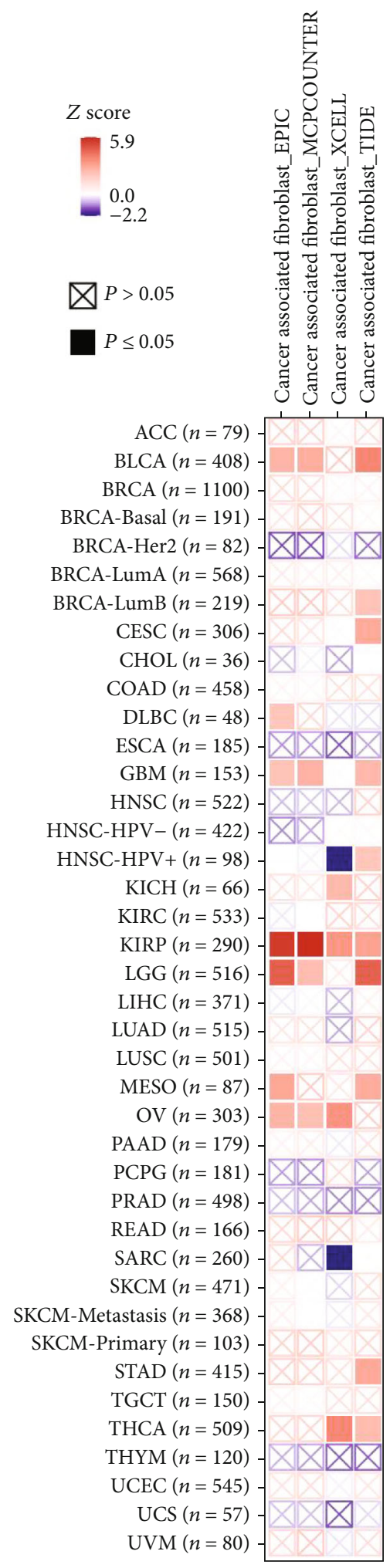

(a)

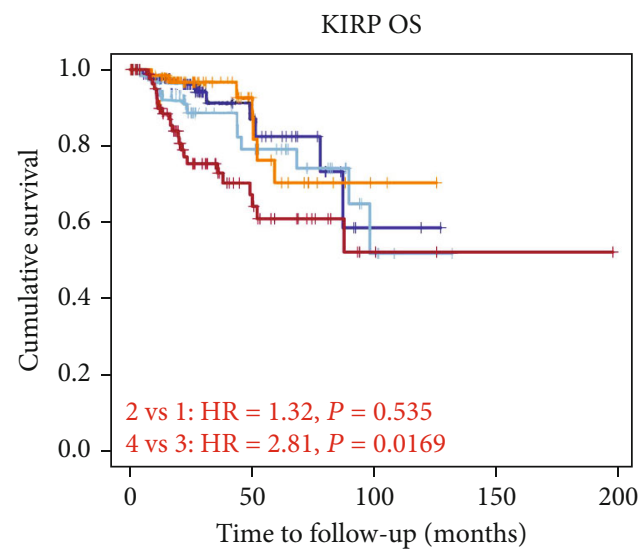

- 1. Low gene expression + Low cancer associated fibroblast_EPIC

- 2. Low gene expression + High cancer associated fibroblast_EPIC

- 3. High gene expression + Low cancer associated fibroblast_EPIC

- 4. High gene expression + High cancer associated fibroblast_EPIC

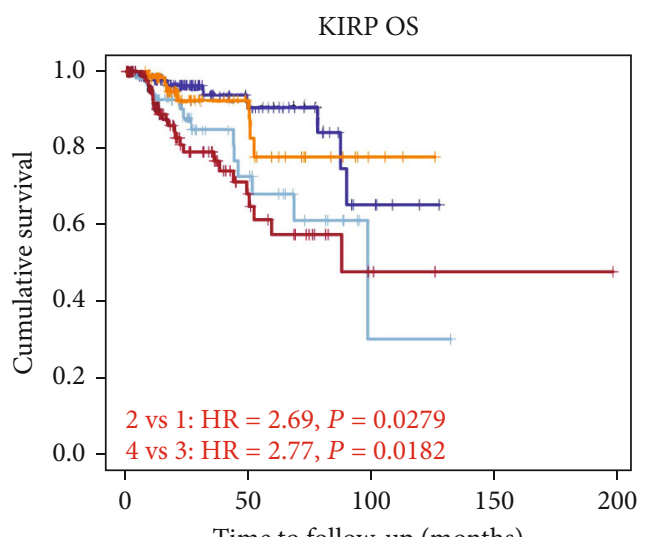

Time to follow-up (months)

- 1. Low gene expression + Low cancer associated fibroblast_MCPCOUNTER 2. Low gene expression + High cancer associated fibroblast_MCPCOUNTER - 3. High gene expression + Low cancer associated fibroblast_MCPCOUNTER - 4. High gene expression + High cancer associated fibroblast_MCPCOUNTER

(b)

Figure 8: Continued. 


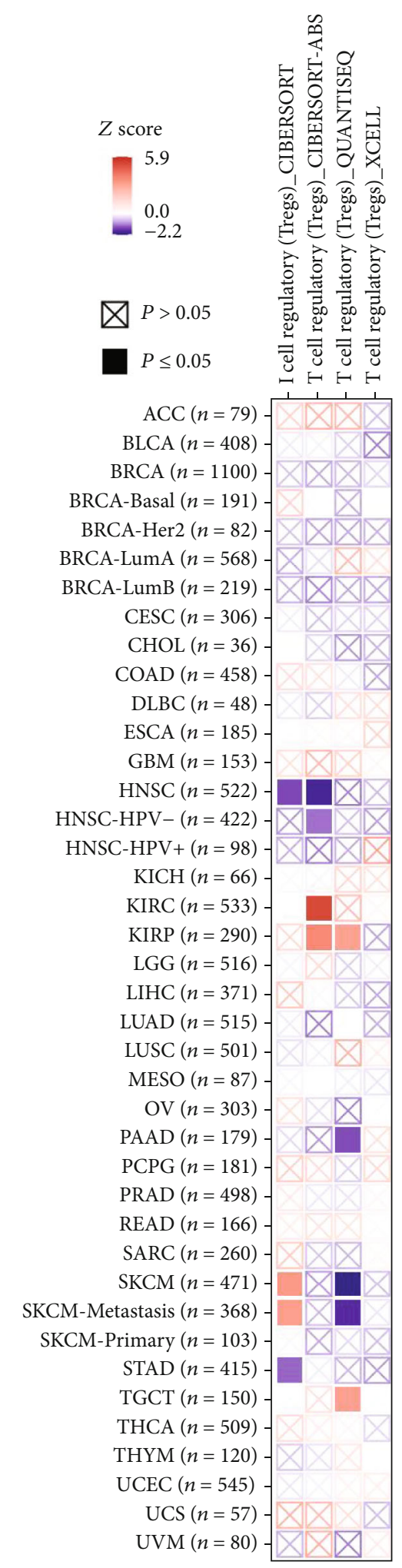

(c)
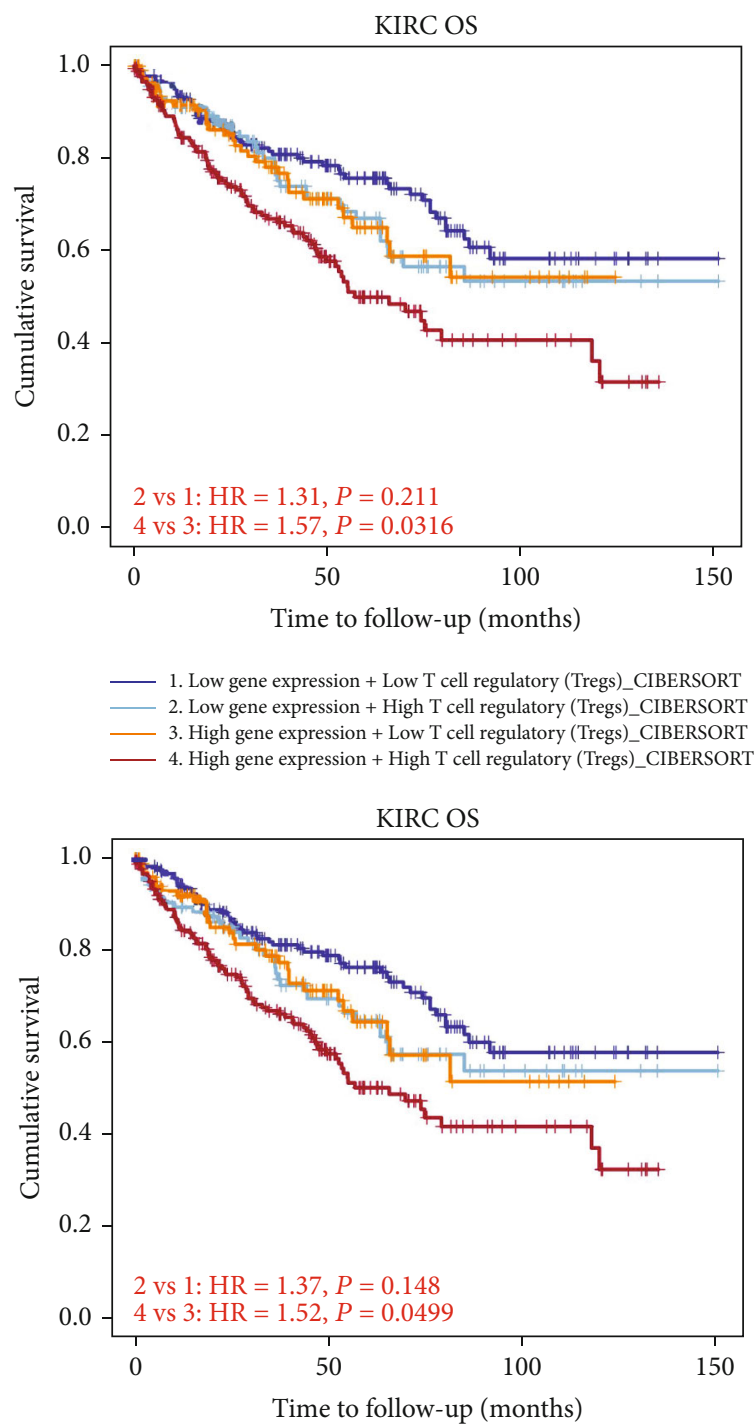

Time to follow-up (months)

- 1. Low gene expression + Low T cell regulatory (Tregs)_CIBERSORT-ABS 2. Low gene expression + High $\mathrm{T}$ cell regulatory (Tregs)_CIBERSORT-ABS 3. High gene expression + Low T cell regulatory (Tregs)_CIBERSORT-ABS — 4. High gene expression + High T cell regulatory (Tregs)_CIBERSORT-ABS

(d)

FIgURE 8: Survival prognosis between WDR62 expression and CAF or Treg cell infiltration. (a) The heat map about OS between WDR62 expression and cancer-associated fibroblasts (CAF). (b) OS between WDR62 expression and cancer-associated fibroblasts (CAF) in KIRP. (c) The heat map about OS between WDR62 expression and T cell regulatory (Treg) cells. (d) OS between WDR62 expression and T cell regulatory (Treg) cells in KIRC. 


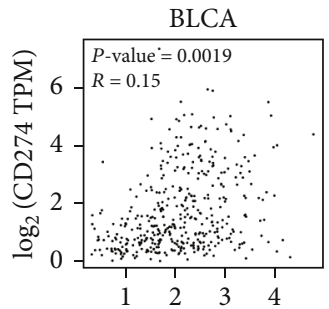

$\log _{2}$ (WDR62 TPM)

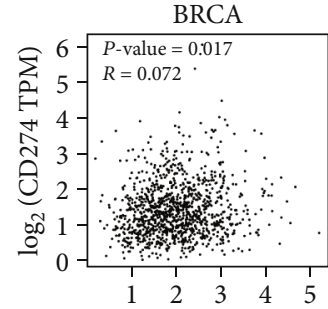

$\log _{2}$ (WDR62 TPM)

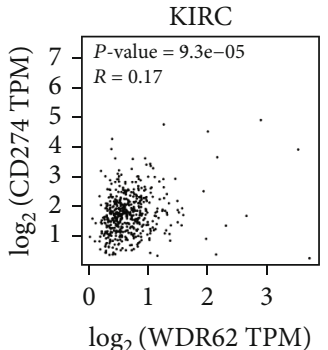

(a)
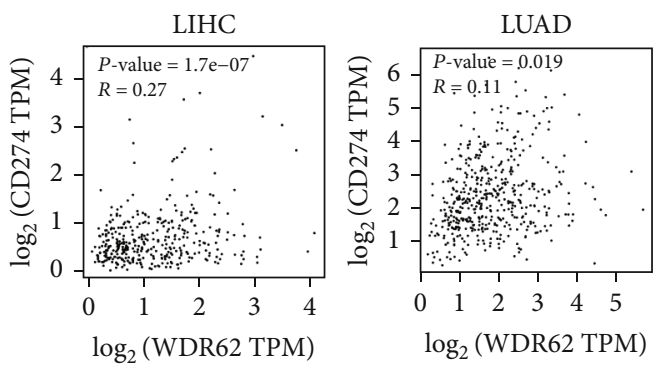

KIRC
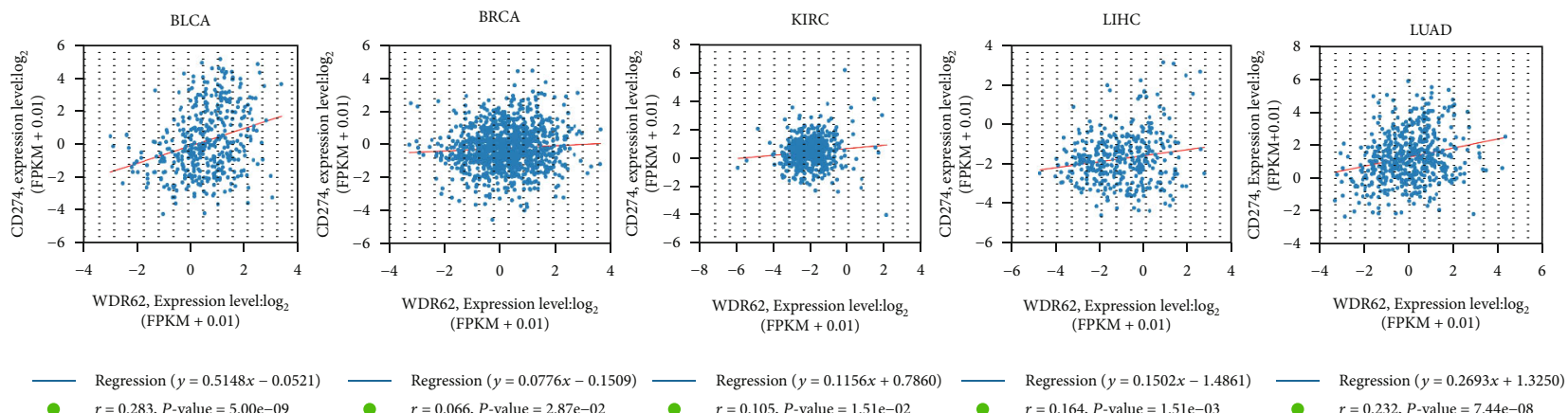

Regression $(y=0.5148 x-0.0521)$
$r=0.283, P$-value $=5.00 \mathrm{e}-09$

Regression $(y=0.0776 x-0.1509)$
$r=0.066, P$-value $=2.87 \mathrm{e}-02$

Regression $(y=0.1156 x+0.7860)$
$\quad r=0.105, P$-value $=1.51 \mathrm{e}-02$

Regression $(y=0.1502 x-1.4861)$
$\quad r=0.164, P$-value $=1.51 \mathrm{e}-03$

$r=0.232, P$-value $=7.44 \mathrm{e}-08$

(b)
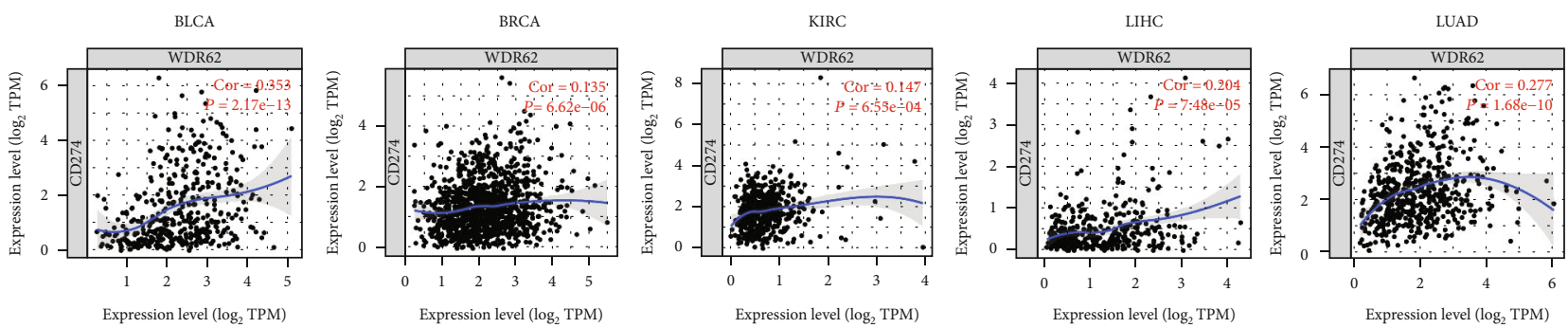

(c)
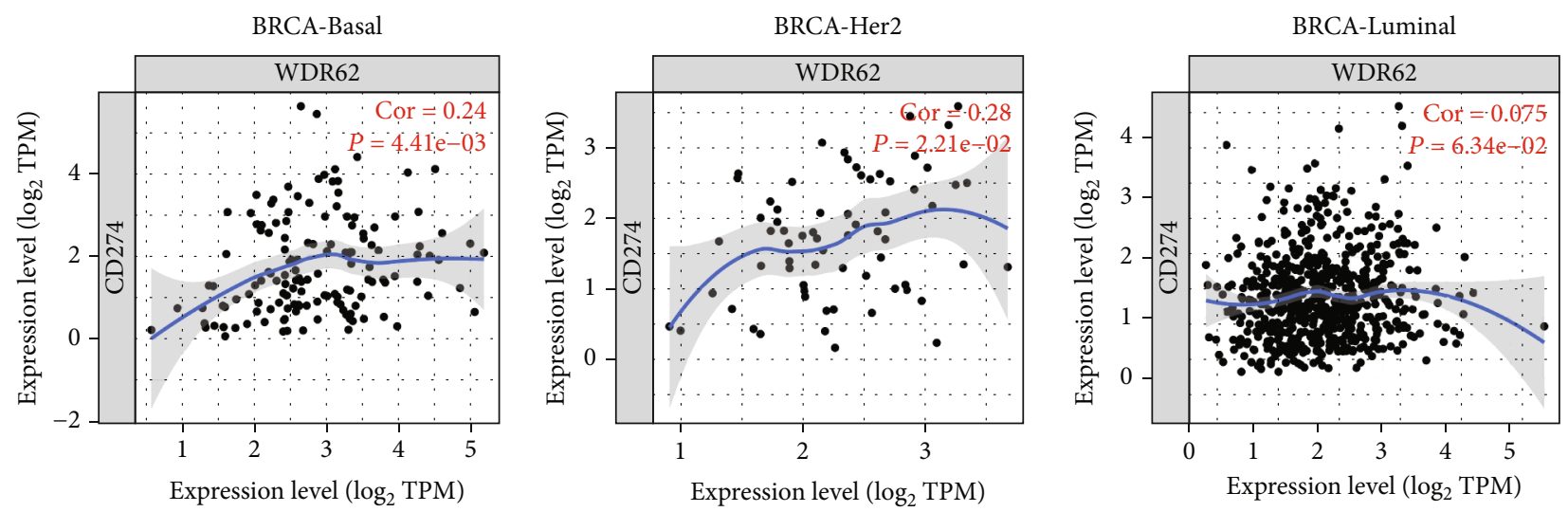

(d)

FIGURE 9: Correlation analysis between WDR62 expression and PD-L1 expression. (a-c) The correlation between WDR62 expression and PD-L1 (CD274) expression was analyzed in the GEPIA2, StarBase, and TIMER databases. (d) The correlation between WDR62 expression and PD-L1 (CD274) expression was analyzed in different subtypes of BRCA. 


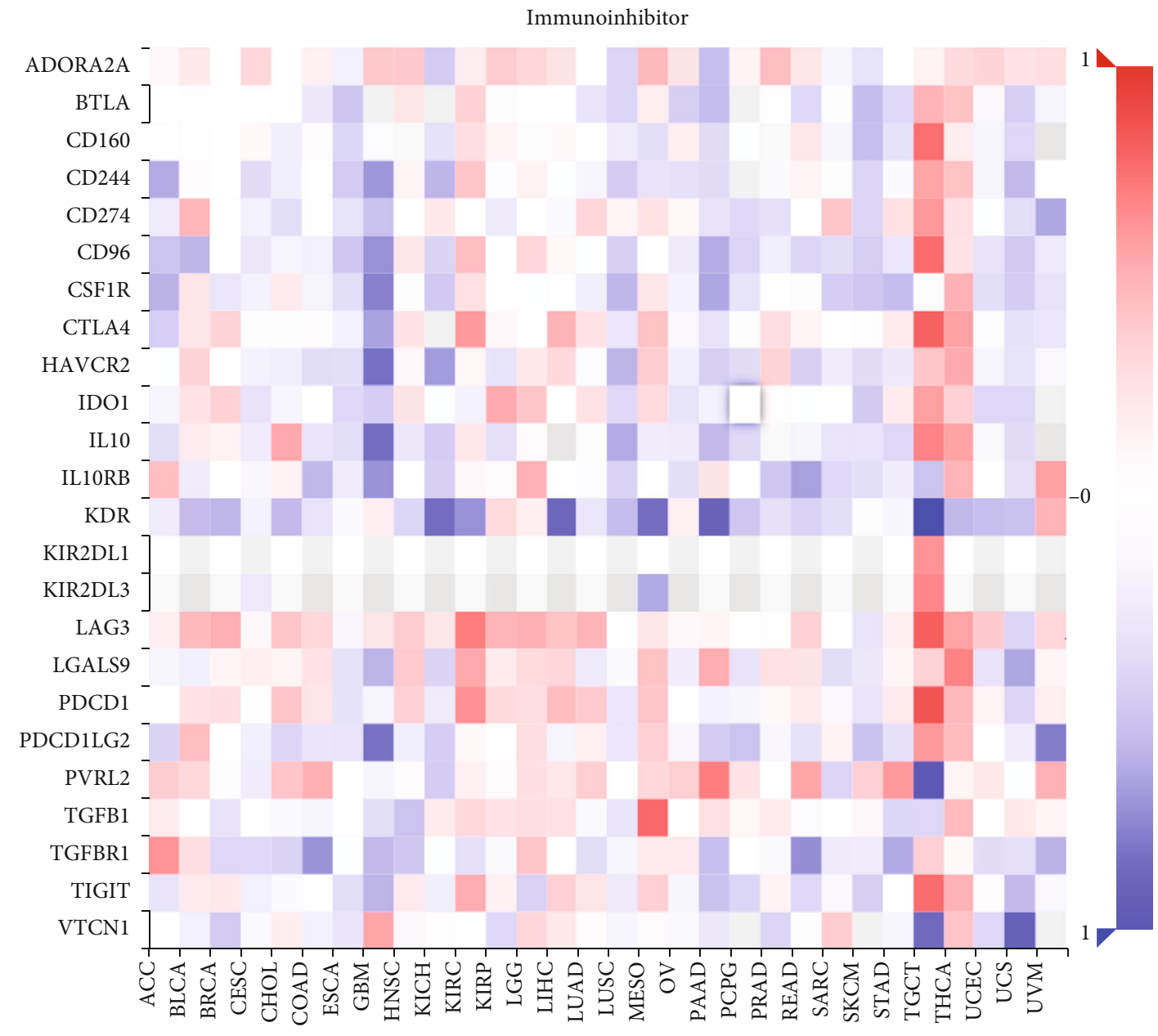

(a)

Figure 10: Continued. 


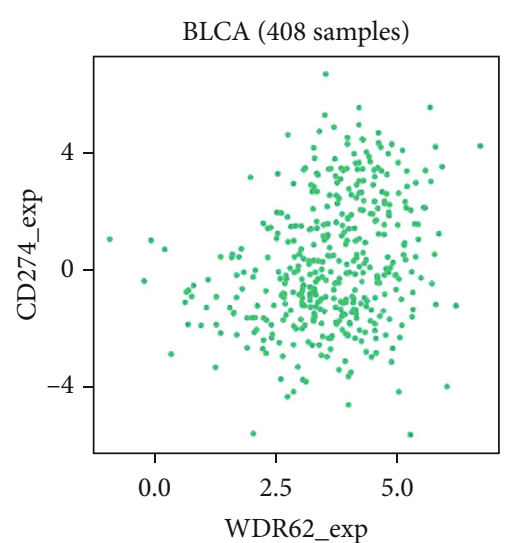

Spearman correlation test: rho $=0.278, P=1.29 \mathrm{e}-08$

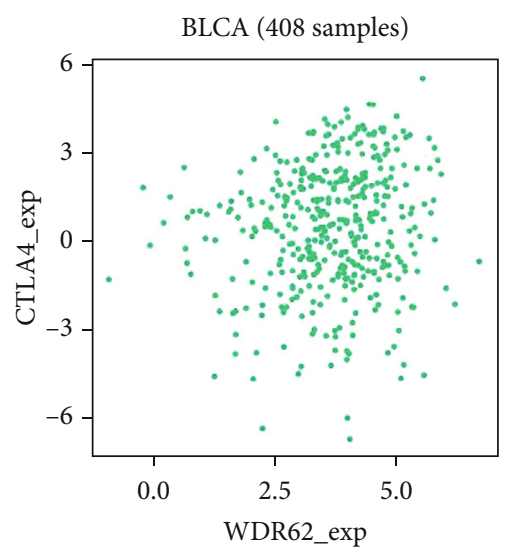

Spearman correlation test: rho $=0.127, P=0.0101$

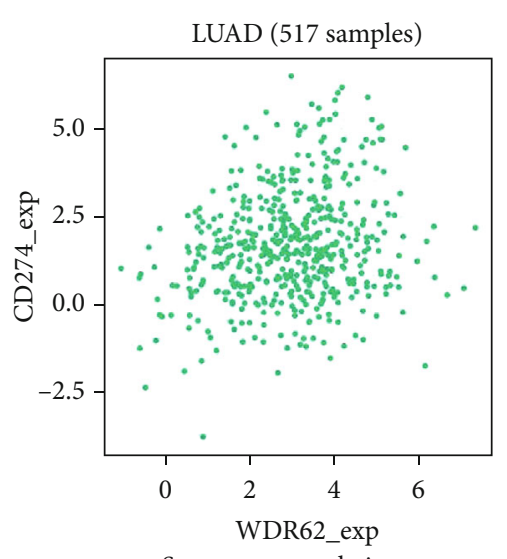

Spearman correlation test: rho $=0.182, P=3.3 \mathrm{e}-05$

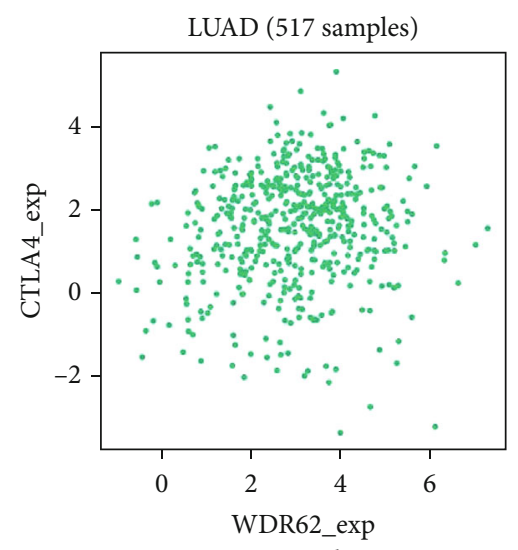

Spearman correlation test: rho $=0.132, P=0.00261$

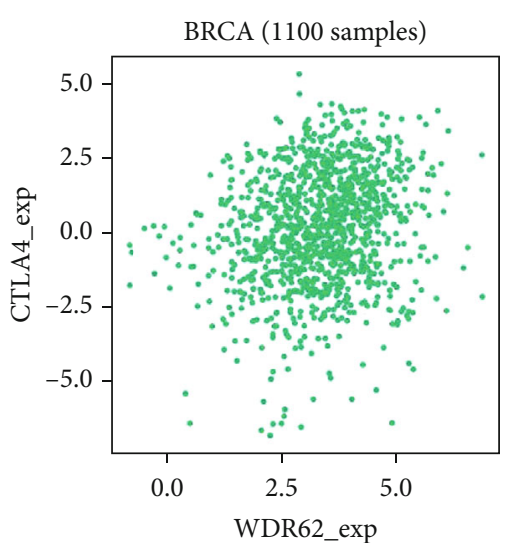

Spearman correlation test: rho $=0.183, P=9.99 \mathrm{e}-10$

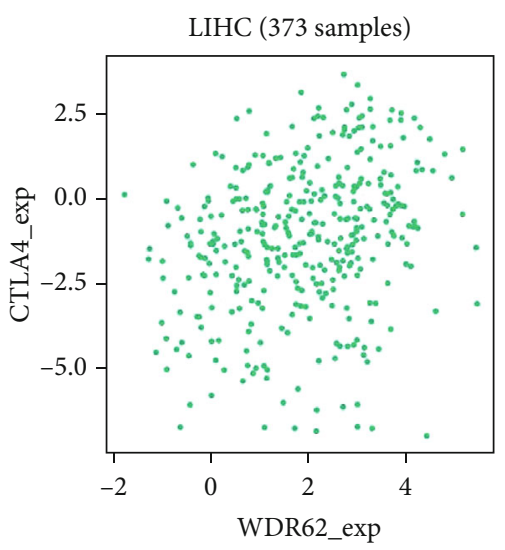

Spearman correlation test: rho $=0.281, P=3.92 \mathrm{e}-08$

(b)

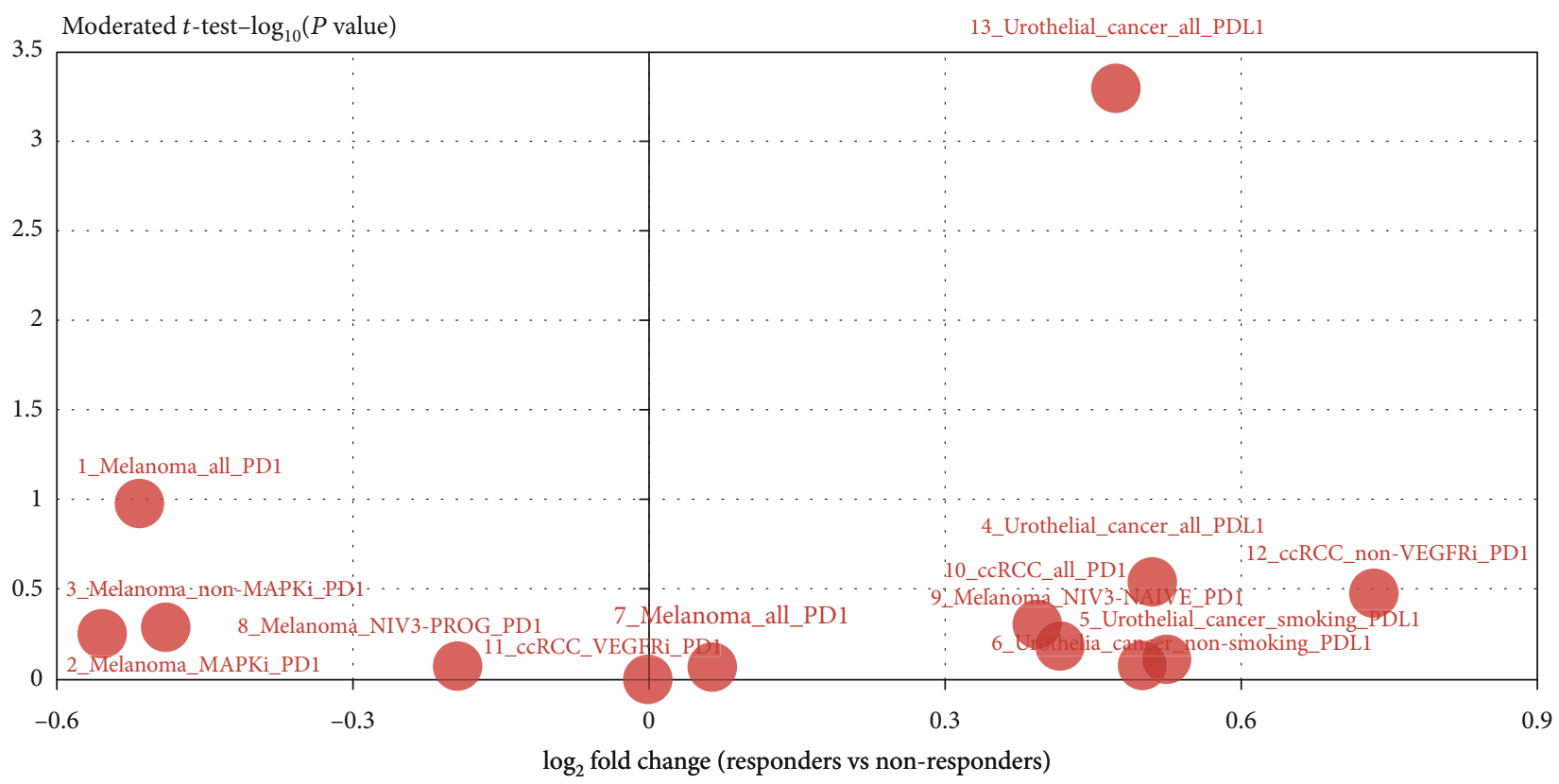

(c)

FIGURE 10: > Association analysis with immunomodulators. (a) The relationship between WDR62 and immunoinhibitors was analyzed by Spearman's correlation analysis via the TISIDB database. (b) The correlation between WDR62 expression and PD-L1 or CTLA4 expression in BLCA, BRCA, LUAD, and LIHC. (c) WDR62 expression difference between responders and nonresponders receiving PD1/PD-L1 inhibitors. 
and efficacy of candidate patients even with the same tumor subtype. Tumor mutation burden (TMB), another biomarker to predict immunotherapy response, is also unsatisfactory which is attributed to its high expense and being timeconsuming [31]. Therefore, new predictive biomarkers for immunotherapy are indispensable for selecting suitable patients and studying innate or acquired resistance. As the most malignant subtype of breast cancer, triple-negative breast cancer (TNBC) received only mild responses in monotherapy with PD1/PD-L1 inhibitors [32]. However, given its higher infiltration level of immune cells, TNBC should have benefited from immunotherapies. Our study presented evidence about the correlation between WDR62 expression and PD-L1 expression in 5 candidate tumors. More importantly, we revealed that WDR62 expression had a marked correlation with PD-L1 expression in TNBC. Therefore, we wonder whether it is suitable to predict the effect of PD1/PD-L1 inhibitors by the detection of WDR62 expression. Based on the TISIDB database, we observed that WDR62 had a significant difference of expression between responders and nonresponders receiving atezolizumab (anti-PD-L1) in urothelial cancer. Collectively, WDR62 could reflect some immune status and indeed could be a predictive biomarker for PD1/PD-L1 inhibitors in some tumor type.

However, our study still has several limits. First, online databases have limitations because different databases produce distinct results due to various sample types and sizes. Moreover, our study showed bioinformatic analysis findings which need further experiments to validate. Additionally, there are many questions to be solved. For example, the underlying molecular mechanism between WDR62 expression and TP53 mutation needs to be explored. How to influence the function of CAF or Treg cells by exosomal WDR62? What about the effect of WDR62 expression on the prediction for immunotherapy in other tumor types? In brief, our first pan-cancer analysis of WDR62 suggested that WDR62 was overexpressed in multiple tumors and closely correlated with poor prognosis and immune regulation, contributing to understanding the oncogenic role of WDR62 across human tumors.

\section{Conclusions}

Our study revealed that WDR62 could serve as a diagnostic and prognostic biomarker for several cancers. Importantly, WDR62 was closely associated with various immune cell infiltration, and to a certain extent, it can predict the effect of immunotherapy in particular PD1/PD-L1 inhibitors. Our pan-cancer study provided useful information on the oncogenic role of WDR62, contributing to further exploring the underlying mechanisms.
Abbreviations
BLCA: Bladder urothelial carcinoma
BP: $\quad$ Biological process
BRCA: Breast invasive carcinoma
CAF: Cancer-associated fibroblasts
CC: Cellular component

DAVID: Database for Annotation, Visualization, and Integrated Discovery

DFS: Disease-free survival

DSS: Disease-specific survival

FP: $\quad$ First progression survival

GEO: Gene Expression Omnibus

GEPIA: Gene Expression Profiling Interactive Analysis

GO: $\quad$ Gene Ontology

HR: Hazard ratio

IARC: International Agency for Research on Cancer

JNK: c-Jun N-terminal kinase

KEGG: Kyoto Encyclopedia of Genes and Genomes

KIRC: Kidney renal clear cell carcinoma

KIRP: Kidney renal papillary cell carcinoma

LIHC: Liver hepatocellular carcinoma

LUAD: Lung adenocarcinoma

MAPK: Mitogen-activated protein kinases

MF: Molecular function

NK: $\quad$ Natural killer T cells

OS: $\quad$ Overall survival

PD1: $\quad$ Programmed cell death 1

PD-L1: Programmed cell death 1 ligand 1

PPI: $\quad$ Protein-protein interaction

PPS: Relapse survival

TCGA: The Cancer Genome Atlas

TIMER: Tumor Immune Evaluation Resource

TM: Tumor microenvironment

TMB: Tumor mutation burden

TNBC: Triple-negative breast cancer

Treg: $\quad$ T cell regulatory

WDR62: WD repeat domain 62 .

\section{Data Availability}

All databases including GEPIA2, TIMER, UALCAN, StarBase, TISIDB, TCGA portal, Kaplan-Meier Plotter, and PrognoScan are freely available as public resources.

\section{Ethical Approval}

TCGA and GEO belong to public databases. The patients involved in the database have obtained ethical approval. Users can download relevant data for free for research and publish relevant articles. Our study is based on open source data, so there are no ethical issues.

\section{Conflicts of Interest}

The authors declare that there is no conflict of interest regarding the publication of this article.

\section{Authors' Contributions}

(i) Jiaqiu Li contributed in conception and design; (II) Xuecheng Zhang contributed in administrative support; (III) Xiaolin Ma and Rui Wang contributed in provision of study materials or patients; (IV) Lihua Zhang contributed in collection and assembly of data; (V) Yulan Bu contributed in data analysis and interpretation; (VI) all authors 
contributed in manuscript writing; (VII) all authors were responsible for the final approval of the manuscript. Yulan $\mathrm{Bu}$ and Lihua Zhang contributed equally to this work.

\section{Acknowledgments}

We acknowledge TCGA and GEO databases for providing their platforms and contributors for uploading their meaningful datasets. This work was supported by the National Nature Science Foundation of China (81902404), Natural Science Foundation of Shandong (ZR2019BH009), and the Project of Shandong Province medical science technology development plan (2017WS403).

\section{Supplementary Materials}

Supplementary 1. S-Figure 1: expression level of WDR62 in different tumors. The expression level of WDR62 in different tumors was analyzed through the GEPIA2 database.

Supplementary 2. S-Figure 2: the correlation between WDR62 expression level and clinical factors in BRCA. WDR62 expression level was analyzed in BRCA tumor stage (A), subclass (B), histologic subtype (C), patient's age (D), patient's race $(\mathrm{E})$, and nodal metastasis status $(\mathrm{F})$ by the UALCAN database.

Supplementary 3. S-Figure 3: correlation between WDR62 expression and survival prognosis of tumors. (A) Prognosis value-OS of KIRC, KIRP, LIHC, and LUAD in the UALCAN database. (B) Prognosis value-OS of KIRC, LIHC, and BRCA-basal in the TIMER database. (C) Prognosis value-OS of KIRC, KIRP, and LIHC in TCGA portal database. (D) The survival value of WDR62 using the PrognoScan database.

Supplementary 4. S-Figure 4: GO and KEGG analysis. (A-D) Based on the WDR62-binding and interacted genes, GO analysis-biological process (BP), molecular function (MF), and cellular component (CC) - and KEGG pathway analysis were performed. (E) The molecular function data in GeneMANIA is shown. (F) WDR62 gene expression profiles in exosome by the exoRBase database.

Supplementary 5. S-Figure 5: correlation analysis between WDR62 expression and immune cell infiltration in 6 candidate tumors-BLCA, BRCA, KIRC, KIRP, LIHC, and LUAD.

Supplementary 6. S-Figure 6: survival prognosis between WDR62 expression and immune cell infiltration. Survival data between WDR62 expression and immune cell infiltration, including CD4+ T cell, CD8+ T cell, macrophage, natural killer (NK) T cells, and regulatory $\mathrm{T}$ cells (Treg), in BLCA, BRCA, KIRC, LIHC, and LUAD using the Kaplan-Meier Plotter.

\section{References}

[1] H. Sung, J. Ferlay, R. L. Siegel et al., "Global cancer statistics 2020: GLOBOCAN estimates of incidence and mortality worldwide for 36 cancers in 185 countries," CA: a Cancer Journal for Clinicians, vol. 71, no. 3, pp. 209-249, 2021.
[2] A. Blum, P. Wang, and J. C. Zenklusen, "SnapShot: TCGAanalyzed tumors,” Cell, vol. 173, no. 2, p. 530, 2018.

[3] E. Clough and T. Barrett, "The Gene Expression Omnibus database," Methods in Molecular Biology, vol. 1418, pp. 93110, 2016.

[4] H. Hayashi and K. Nakagawa, "Combination therapy with PD1 or PD-L1 inhibitors for cancer," International Journal of Clinical Oncology, vol. 25, no. 5, pp. 818-830, 2020.

[5] R. W. Jenkins, D. A. Barbie, and K. T. Flaherty, "Mechanisms of resistance to immune checkpoint inhibitors," British Journal of Cancer, vol. 118, no. 1, pp. 9-16, 2018.

[6] Y. Zhang, Y. Tian, J. J. Yu et al., "Overexpression of WDR62 is associated with centrosome amplification in human ovarian cancer," J Ovarian Res, vol. 6, no. 1, p. 55, 2013.

[7] S. Zeng, Y. Tao, J. Huang et al., "WD40 repeat-containing 62 overexpression as a novel indicator of poor prognosis for human gastric cancer," European Journal of Cancer, vol. 49, no. 17, pp. 3752-3762, 2013.

[8] Z. Tang, B. Kang, C. Li, T. Chen, and Z. Zhang, "GEPIA2: an enhanced web server for large-scale expression profiling and interactive analysis," Nucleic Acids Research, vol. 47, no. W1, pp. W556-W560, 2019.

[9] T. Li, J. Fan, B. Wang et al., "TIMER: a web server for comprehensive analysis of tumor-infiltrating immune cells," Cancer Research, vol. 77, no. 21, pp. e108-e110, 2017.

[10] B. Li, E. Severson, J. C. Pignon et al., "Comprehensive analyses of tumor immunity: implications for cancer immunotherapy," Genome Biology, vol. 17, no. 1, p. 174, 2016.

[11] D. S. Chandrashekar, B. Bashel, S. A. H. Balasubramanya et al., "UALCAN: a portal for facilitating tumor subgroup gene expression and survival analyses," Neoplasia, vol. 19, no. 8, pp. 649-658, 2017.

[12] J. H. Li, S. Liu, H. Zhou, L. H. Qu, and J. H. Yang, "starBase v2.0: decoding miRNA-ceRNA, miRNA-ncRNA and protein-RNA interaction networks from large-scale CLIPSeq data," Nucleic Acids Research, vol. 42, no. D1, pp. D92D97, 2014.

[13] B. Ru, C. N. Wong, Y. Tong et al., “TISIDB: an integrated repository portal for tumor-immune system interactions," Bioinformatics, vol. 35, no. 20, pp. 4200-4202, 2019.

[14] S. Xu, Y. Feng, and S. Zhao, "Proteins with evolutionarily hypervariable domains are associated with immune response and better survival of basal-like breast cancer patients," Computational and Structural Biotechnology Journal, vol. 17, pp. 430-440, 2019.

[15] A. Nagy, A. Lanczky, O. Menyhart, and B. Gyorffy, "Validation of miRNA prognostic power in hepatocellular carcinoma using expression data of independent datasets," Scientific Reports, vol. 8, no. 1, p. 9227, 2018.

[16] H. Mizuno, K. Kitada, K. Nakai, and A. Sarai, "PrognoScan: a new database for meta-analysis of the prognostic value of genes," BMC Medical Genomics, vol. 2, no. 1, p. 18, 2009.

[17] D. Szklarczyk, A. L. Gable, D. Lyon et al., "STRING v11: protein-protein association networks with increased coverage, supporting functional discovery in genome-wide experimental datasets," Nucleic Acids Research, vol. 47, no. D1, pp. D607D613, 2019.

[18] S. Mostafavi, D. Ray, D. Warde-Farley, C. Grouios, and Q. Morris, "Gene MANIA: a real-time multiple association network integration algorithm for predicting gene function," Genome Biology, vol. 9, Supplement 1, p. S4, 2008. 
[19] D. Warde-Farley, S. L. Donaldson, O. Comes et al., "The GeneMANIA prediction server: biological network integration for gene prioritization and predicting gene function," Nucleic Acids Research, vol. 38, supplement 2, pp. W214-W220, 2010.

[20] D. W. Huang, B. T. Sherman, and R. A. Lempicki, "Systematic and integrative analysis of large gene lists using DAVID bioinformatics resources," Nature Protocols, vol. 4, no. 1, pp. 44-57, 2009.

[21] A. K. Nicholas, M. Khurshid, J. Desir et al., "WDR62 is associated with the spindle pole and is mutated in human microcephaly," Nature Genetics, vol. 42, no. 11, pp. 1010-1014, 2010.

[22] M. A. Bogoyevitch, Y. Y. Yeap, Z. Qu et al., "WD40-repeat protein 62 is a JNK-phosphorylated spindle pole protein required for spindle maintenance and timely mitotic progression," Journal of Cell Science, vol. 125, no. Partt 21, pp. 5096-5109, 2012.

[23] T. W. Yu, G. H. Mochida, D. J. Tischfield et al., "Mutations in WDR62, encoding a centrosome-associated protein, cause microcephaly with simplified gyri and abnormal cortical architecture," Nature Genetics, vol. 42, no. 11, pp. 1015-1020, 2010.

[24] K. Shinmura, H. Kato, Y. Kawanishi et al., "WDR62 overexpression is associated with a poor prognosis in patients with lung adenocarcinoma," Molecular Carcinogenesis, vol. 56, no. 8, pp. 1984-1991, 2017.

[25] S. Sugita, H. Yoshino, M. Yonemori et al., "Tumor-suppressive microRNA-223 targets WDR62 directly in bladder cancer," International Journal of Oncology, vol. 54, no. 6, pp. 22222236, 2019.

[26] E. Prinz, S. Aviram, and A. Aronheim, "WDR62 mediates TNF $\alpha$-dependent JNK activation via TRAF2-MLK3 axis," Molecular Biology of the Cell, vol. 29, no. 20, pp. 2470-2480, 2018.

[27] A. Steven and B. Seliger, "The role of immune escape and immune cell infiltration in breast cancer," Breast Care (Basel), vol. 13, no. 1, pp. 16-21, 2018.

[28] W. H. Fridman, J. Galon, M. C. Dieu-Nosjean et al., "Immune infiltration in human cancer: prognostic significance and disease control," in Current Topics in Microbiology and Immunology 344, pp. 1-24, Springer, Verlag Berlin Heidelberg, 2011.

[29] R. Xu, A. Rai, M. Chen, W. Suwakulsiri, D. W. Greening, and R. J. Simpson, "Extracellular vesicles in cancer - implications for future improvements in cancer care," Nature Reviews. Clinical Oncology, vol. 15, no. 10, pp. 617-638, 2018.

[30] J. R. Brahmer, S. S. Tykodi, L. Q. Chow et al., "Safety and activity of anti-PD-L1 antibody in patients with advanced cancer," The New England Journal of Medicine, vol. 366, no. 26, pp. 2455-2465, 2012.

[31] G. Cyriac and L. Gandhi, "Emerging biomarkers for immune checkpoint inhibition in lung cancer," Seminars in Cancer Biology, vol. 52, no. Partt 2, pp. 269-277, 2018.

[32] H. Zhu, C. Du, M. Yuan et al., "PD-1/PD-L1 counterattack alliance: multiple strategies for treating triple- negative breast cancer," Drug Discovery Today, vol. 25, no. 9, pp. 1762-1771, 2020. 